\title{
Recent developments in predictive uncertainty assessment based on the model conditional processor approach
}

\author{
G. Coccia ${ }^{1,2}$ and E. Todini ${ }^{1}$ \\ ${ }^{1}$ Department of Earth and Geo-Environmental Sciences, University of Bologna, Bologna, Italy \\ ${ }^{2}$ Idrologia e Ambiente s.r.l., Riviera di Chiaia 72, 80122, Napoli, Italy
}

Received: 17 November 2010 - Published in Hydrol. Earth Syst. Sci. Discuss.: 6 December 2010

Revised: 20 October 2011 - Accepted: 23 October 2011 - Published: 28 October 2011

\begin{abstract}
The work aims at discussing the role of predictive uncertainty in flood forecasting and flood emergency management, its relevance to improve the decision making process and the techniques to be used for its assessment.

Real time flood forecasting requires taking into account predictive uncertainty for a number of reasons. Deterministic hydrological/hydraulic forecasts give useful information about real future events, but their predictions, as usually done in practice, cannot be taken and used as real future occurrences but rather used as pseudo-measurements of future occurrences in order to reduce the uncertainty of decision makers. Predictive Uncertainty (PU) is in fact defined as the probability of occurrence of a future value of a predictand (such as water level, discharge or water volume) conditional upon prior observations and knowledge as well as on all the information we can obtain on that specific future value from model forecasts. When dealing with commensurable quantities, as in the case of floods, PU must be quantified in terms of a probability distribution function which will be used by the emergency managers in their decision process in order to improve the quality and reliability of their decisions.
\end{abstract}

After introducing the concept of PU, the presently available processors are introduced and discussed in terms of their benefits and limitations. In this work the Model Conditional Processor (MCP) has been extended to the possibility of using two joint Truncated Normal Distributions (TNDs), in order to improve adaptation to low and high flows.

The paper concludes by showing the results of the application of the MCP on two case studies, the Po river in Italy and the Baron Fork river, OK, USA. In the Po river case the

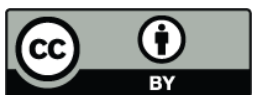

Correspondence to: G. Coccia (gabriele.coccia@idrologiaeambiente.com) data provided by the Civil Protection of the Emilia Romagna region have been used to implement an operational example, where the predicted variable is the observed water level. In the Baron Fork River example, the data set provided by the NOAA's National Weather Service, within the DMIP 2 Project, allowed two physically based models, the TOPKAPI model and TETIS model, to be calibrated and a data driven model to be implemented using the Artificial Neural Network. The three model forecasts have been combined with the aim of reducing the PU and improving the probabilistic forecast taking advantage of the different capabilities of each model approach.

\section{Introduction}

\subsection{Decision making under uncertainty}

In the last decades, the interest in assessing uncertainty in models forecasts has grown exponentially within the scientific communities of meteorologists and hydrologists. In particular, the introduction of the Hydrological Uncertainty Processor (Krzysztofowicz, 1999; Krzysztofowicz and Kelly, 2000), aimed at assessing the predictive uncertainty in hydrological forecasts, has created the basis for the estimation of flood predictive uncertainty.

Flood emergency management requires adopting operational decisions in real time that may lead to dramatic consequences (economical losses, casualties, etc.). The hardest obstacle the managers have to deal with is the uncertainty on the future evolution of events. Decision theory (De Groot, 1970; Raiffa and Schlaifer, 1961) studied this problem and provided the most appropriate solutions for taking decisions under uncertainty. This approach consists in minimizing the

Published by Copernicus Publications on behalf of the European Geosciences Union. 
expected value of a utility function $U(y)$ representing the losses, or more in general the subjective manager perception of them, as a function of a predictand that will occur at a future time (such as a future discharge or water stage in a cross section). This quantity is unknown at the time of the decision $\left(t_{0}\right)$ and the aim of forecasting is to assess its probability of occurrence, in terms of a predictive uncertainty probability density function.

In the case of flood forecasting, predictive uncertainty can be defined as the uncertainty that a decision maker has on the future evolution of a predictand that he uses to make a specific decision.

In order to fully understand and to appreciate what is actually meant by predictive uncertainty, it is necessary to realize that what will cause the flooding damages is the actual future realization of the discharge and/or the water level that will occur, not the prediction generated by a forecasting model; in other words the damages will occur when the actual water level $y_{t}$ and certainly not if the prediction $\hat{y}_{t}$ will overtop the dyke level $y_{\mathrm{D}}$ (Todini, 2009). Therefore a utility/damage function at any future time $\left(t>t_{0}\right)$ must be expressed as a function of the actual level that will occur at time $t$

$$
\begin{cases}U\left(y_{t}\right)=0 & \forall y_{t} \leq y_{\mathrm{D}} \\ U\left(y_{t}\right)=g\left(y_{t}-y_{\mathrm{D}}\right) & \forall y_{t}>y_{\mathrm{D}}\end{cases}
$$

where $g(\cdot)$ represents a generic function relating the cost of damages and losses to the future, albeit unknown water stage $y_{t}$. In this case the manager, according to the decision theory (De Groot, 1970; Raiffa and Schlaifer, 1961), must take his decisions on the basis of the expected utility $E\left\{U\left(y_{t}\right)\right\}$. This value can be estimated only if the probability density function of the future event is known, and it can be written as

$$
E\left\{U\left(y_{t}\right)\right\}=\int_{0}^{+\infty} U\left(y_{t}\right) f\left(y_{t}\right) d y_{t}
$$

where $f\left(y_{t}\right)$ is the probability density expressing our incomplete knowledge (in other words our uncertainty) on the future value that will occur. This density, which can be estimated from historical data, is generally too broad because it lacks the conditionality on the current events. This is why it is essential to improve this historical probability distribution function by more realistically using one or more hydrological models able to summarize all the available information (like the rain forecast, the catchment geomorphology, the state of the river at the moment of the forecast, etc...) and to provide a more informative density $f\left(y_{t} \mid \hat{y}_{t \mid t_{0}}\right)$, which expresses our uncertainty on the future predictand value after knowing the models' forecasts issued at time $t_{0}$, namely $\hat{y}_{t \mid t_{0}}=\left[\hat{y}_{1_{t \mid t_{0}}}, \hat{y}_{2_{t \mid t_{0}}}, \ldots, \hat{y}_{M_{t \mid t_{0}}}\right.$, where $M$ is the number of forecasting models. Equation (2) can now be rewritten as

$E\left\{U\left(y_{t} \mid \hat{\boldsymbol{y}}_{t \mid t_{0}}\right)\right\}=\int_{0}^{+\infty} U\left(y_{t}\right) f\left(y_{t} \mid \hat{\boldsymbol{y}}_{t \mid t_{0}}\right) d y_{t}$
The probability distribution function $f\left(y_{t} \mid \hat{\boldsymbol{y}}_{t \mid t_{0}}\right)$ represents the PU, hereafter denominated $f(y \mid \hat{\boldsymbol{y}})$ for sake of simplicity.

In this paper some existing uncertainty processors will be breafly discussed, focusing on the Model Conditional Processor (Todini, 2008), with particual attention to the error heteroscedasticity and the models combination, providing a solution to tackle them.

\subsection{Choice of the predictand}

An important issue in PU assessment is the choice of the predictand. As mentioned in the previuos section, PU deals with the actual value of the variable to be predicted. To this end, it is well known that measurements are always affected by errors that should be taken into account. However substituting the observed value for the actual value is a common procedure in PU assessment even if observations do not coincide with reality. A discussion about the choice of the predictand will be carried out in this section.

It is worth to take into account the following four remarks:

- Water level measurements are affected by relatively small errors (with standard error of the order of 2$3 \mathrm{~cm}$ ); although care should be taken to account for possible non stationarities in the records, it is psychologically fundamental to use them as measures unaffected by measurement errors both because flood decisions have always been essentially based on these measures and because their errors have very small effect on the decisions compared to the larger effects of the other sources of uncertainty.

- Discharge measurements are generally unavailable in real time, although there is a recent tendency to use microwave surface velocity measurements in combination with the water stage, which could improve discharge estimates in real time.

- Classical discharge estimates, based on water level measurements and steady state rating curves, are affected by errors that may reach $30 \%$ (Di Baldassare and Montanari, 2009). One major source of errors is due to extrapolation beyond the range of observations. A second major source is due to the presence of loops in the level-discharge relation, which are not represented by the steady state rating curve, unless modified by using correcting formulas, such as the Jones formula or others (Dottori et al., 2009).

- Rainfall-runoff model forecasts are essentially based on discharges, while flood routing model forecasts can be obtained both in terms of water levels and/or discharges. In any case they are affected by a wide range of errors.

Therefore, when dealing with real time flood forecasting, the filtered water level is in theory the most appropriate quantity to be used as the predictand. Please note that we are 
here talking of a filtered quantity because the real occurrence will never be known, but one can reduce the measurement errors by using filtering techniques aimed at reducing measurement errors, such as for instance the classical Kalman Filtering technique (Kalman, 1960; Kalman and Bucy, 1961). Nonetheless, in practice, the observed water levels can be considered the best operational quantity to be used as predictand: the errors are small and the decision makers degree of belief is very high, while this is not so for the filtered quantities that are estimated and not measured. Therefore, whenever possible, and in particular when dealing with flood warning, one should use the observed water levels as the predictand to be used in any flood predictive uncertainty processor.

In case that water levels are not available or when one needs to predict inflows to a reservoir or a water detention area, where the flood volumes are the best decision variable, corrected and filtered discharges should be used. In other words, prior to use discharges as predictands for the calibration of the hydrological uncertainty processors, their improved estimates must be produced both by accounting for the shape of the cross section and by taking into account the loop formation in the rating curve. This will eliminate most of the water level dependent biases, while the elimination of the random errors must be approached by filtering techniques.

In terms of predictors (i.e. the variables used to condition the PU), when available from a flood routing model, the best choice would be the forecasted water levels. Otherwise it is possible either to convert the predicted discharges into predicted water levels using a corrected rating curve, as mentioned above, or just to directly use the predicted discharges, since the effect of the conversion errors from discharge to levels, may affect the order of the predicted variables. This is what essentially dominates the Normal Quantile Transform (NQT), which is the basis of most of the uncertainty processors (Van der Waerden, 1952, 1953a,b).

\subsection{The probabilistic threshold paradigm}

Today, similarly to what was done for more than a century, in order to trigger their decisions, the majority of water authorities involved in flood emergency management prepare their plans on the basis of pre-determined water depths or thresholds ranging from the warning water level to the flooding level. Decisions, and consequent actions, are then taken as soon as a real time measure of the water stage overtops one of these thresholds. This approach, which is correct and sound in the absence of flood forecasting models, is a way of anticipating events on the basis of water level measures (in the cross sections of interest or in upstream cross sections), but can only be effective on very large rivers where the time lag between the overtopping of the warning and the flooding levels is sufficiently large to allow for the implementation of the planned flood relief strategies and interventions. Given that all the water stage measures are affected by relatively small errors $(2-3 \mathrm{~cm})$, they can be, and have been, considered as deterministic.

Unfortunately, the advent and the operational use of real time flood forecasting models, has not changed this approach, which has been the cause of several unsatisfactory results. Today, the flood managers compare forecasts, and not the actual measurements, to the different threshold levels; this is obviously done in order to further anticipate decisions by taking advantage of the prediction time horizon. Unfortunately, by doing so the forecasts are implicitly assumed to be deterministic, which is not the case since they represent virtual reality and are affected by prediction errors, which magnitude is by far larger than that of the measurement errors.

More recently, the concept of predictive uncertainty has changed this approach. This uncertain nature of forecasts, opposed to the higher accuracy of measurements, requires the definition of probabilistic thresholds, defined in terms of the probability of flooding taken at different probability levels, instead of the definition of deterministic threshold values. Using the probabilistic thresholds, the same predicted water level may have different meaning owing to the reliability of prediction. In other words, the same forecast may or may not trigger the decision of issuing a warning or evacuating an area, conditionally to its assessed level of uncertainty. More uncertain forecasts need necessarily to be treated more cautiously than more reliable ones; in fact, uncertain lower water stage forecasts could then trigger a protective measure, whereas higher, albeit more accurate water stage forecasts, would not. As can be seen from the Fig. 1, for the same expected value (the horizontal dashed line) a better forecast (Model A), characterised by a narrower predictive density, will show a smaller probability of exceeding the flooding level when compared to a worse one (Model B). This property can be also looked at from an alternative perspective, as shown in Fig. 2 the same flooding probability corresponds to lower expected values as the spread of PU increases. This implies that if a probabilistic threshold is defined instead of a deterministic threshold level, when the PU is larger the decision maker must be more cautious and would be advised to issue an alert even when, looking at the expected value of the forecast, he would not think of issuing it, because he may regard it as being too low.

\section{Existing approaches}

In the following sections the Hydrological Uncertanty Processor (Krzysztofowicz, 1999), the Bayesian Model Averaging (Raftery, 1993; Raftery et al., 2005; Vrugt and Robinson, 2007) and the Quantile Regression (Koenker, 2005) will be briefly discussed, but many other bayesian processors exist, such as the one introduced by Kennedy and O'Hagan (2001). 


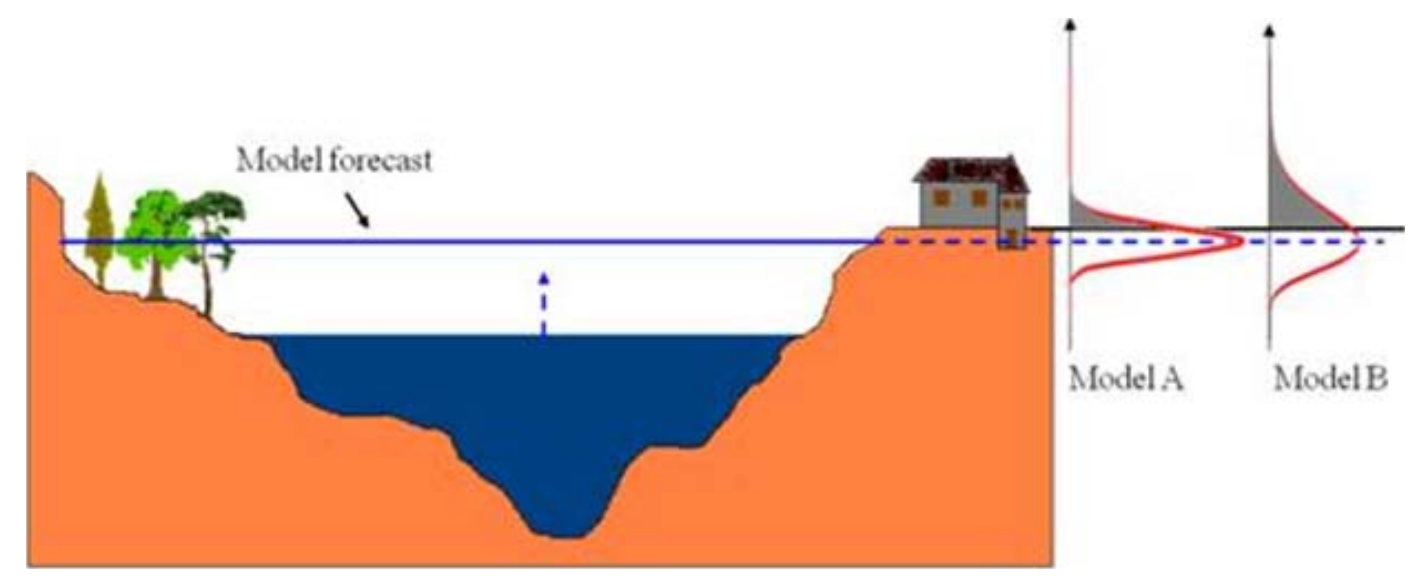

Fig. 1. Probability of exceeding the dyke level for the same expected value, forecasted by models with different reliability.

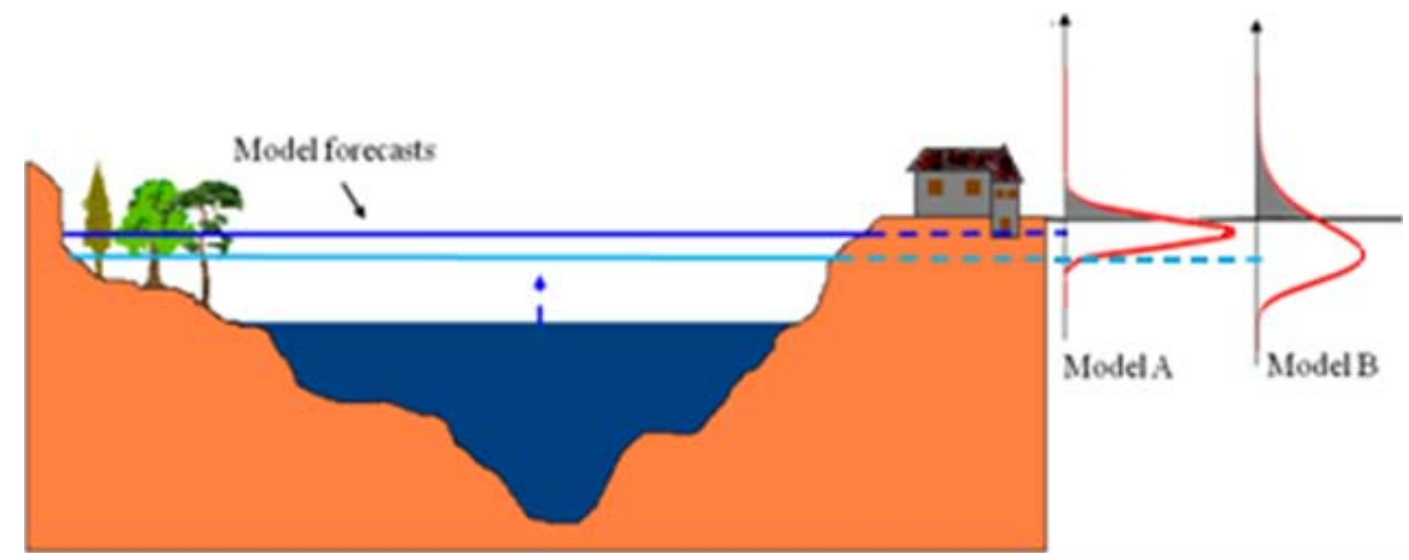

Fig. 2. Comparison between the expected value provided by models with different reliability when the probability of exceeding the dyke level is the same for all the models.

\subsection{Hydrological uncertainty processor}

Krzysztofowicz (1999) introduced a Bayesian processor, the Hydrological Uncertainty Processor (HUP) which aims at estimating the predictive uncertainty given a set of historical observations and a hydrological model prediction. The HUP was developed around the idea of converting both observations and model predictions into a normal space by means of the NQT in order to derive the joint distribution and the predictive conditional distribution from a treatable multivariate distribution. In practice, as described in Krzysztofowicz (1999), after converting the observations and the model forecasts available for the historical period into the normal space, the HUP combines the prior predictive uncertainty (in this case derived using an autoregressive model) with a Likelihood function in order to obtain the posterior density of the predictand conditional to the model forecasts. From the normal space this conditional density is finally reconverted into the real space in order to provide the predictive probability density.
The introduction of HUP generated a positive impact on the hydrological community, in fact Krzysztofowicz (1999) was the first in hydrological forecasting who clarified the concept of predictive uncertainty. Nonetheless, HUP has three major limitations. The first one relates to the fact that only one model at a time can be used in HUP, which has not yet been extended to multi model forecasts. Moreover the used prior autoregressive (AR) model frequently tends to be inadequate to represent the predictand, as for instance in the case of a flood routing problem where the AR model is adequate for representing the recession but not the rising limb of the flood wave. Finally, the HUP procedure implies the independence of the AR model errors from those deriving from the used prediction model, which is not guaranteed due to the fact that both models tend to be highly correlated to the observations, which inevitably induces a level of correlation among them. 


\subsection{Bayesian model averaging}

Introduced by Raftery (1993), Bayesian Model Averaging (BMA) has gained a certain popularity in the latest years. The scope of Bayesian Model Averaging is correctly formulated in that it aims at assessing the mean and variance of any future value of the predictand conditional upon several model forecasts. Differently from the HUP assumptions, in BMA all the models (including the AR prior model) are similarly considered as alternative models. Raftery et al. (2005) developed the approach on the assumption that the predictand as well as the model forecasts were approximately normally distributed, while Vrugt and Robinson (2007) relaxed this hypothesis and showed how to apply the BMA to Log-normal and Gamma distributed variables. In practice the Bayesian Inference problem, namely the need for estimating a posterior density for the parameters, is overcome in the BMA by estimating a number of weights via a constrained optimization problem. Once the weights have been estimated, BMA allows to estimate the mean and the variance of the predictand conditional upon several models at the same time.

The original BMA, as introduced by Raftery (1993), has shown several problems. First of all, as pointed out by Vrugt and Robinson (2007), the original assumption of approximately normally distributed errors, is not appropriate for representing highly skewed quantities such as water discharges or water levels in rivers. Therefore one must either relax this hypothesis, as done by Vrugt and Robinson (2007) who applied the BMA to Log-normal and Gamma distributed variables or to convert the original in the normal space once again using the NQT, as done in Todini (2008). Another problem, which emerges from the application of BMA is the use of the "expectation-maximization" (EM) algorithm (Dempster et al., 1977) proposed by Raftery et al. (2005), which was not found to properly converge to the maximum of the likelihood. To overcome this problem, one can either use sophisticated, complex optimization tools such as the SCEM-UA (Vrugt et al., 2003) or, as proposed by Todini (2008), a simple and original constrained Newton-Raphson approach, which converges in a very limited number of iterations.

\subsection{Quantile regression}

The latest uncertainty processors approaches tackle the problem of the heteroscedasticity of the errors often present in hydrological modelling. All the previously described techniques imply homoscedasticity of the error variance, which is assumed to be independent from the magnitude of the observed or forecasted values, but in real cases this assumption leads to a lack of accuracy.

Recently, in order to overcome this problem, the Quantile Regression (Koenker, 2005) was used (Weerts et al., 2011). The Quantile Regression (QR) approach tries to represent the error heteroscedasticity identifying a linear or non-linear variation of the quantiles of the PU as a function of the model forecast magnitude. This technique allows all the desired quantiles of the PU to be assessed in the normal space and then reconverted by means of the inverse NQT to the real space. In the linear case, the $\tau$-th sample quantile is computed solving the Eq. (4), from which is possible to identify the parameters $a_{\tau}$ and $b_{\tau}$ which defines the linear regression for the $\tau$-th quantile.

$\min _{a_{\tau}, b_{\tau} \in R} \sum_{i=1}^{n} \rho_{\tau}\left(\eta-a-b_{\tau} \cdot \hat{\eta}\right)$

where

$\rho_{\tau}(x)=\left\{\begin{array}{lll}x \cdot(\tau-1) & \text { if } \quad x<0 \\ x \cdot \tau & \text { if } \quad x \geq 0\end{array}\right.$

The problem is correctly formulated and allows each quantile of the PU to be computed, but it requires the estimation of at least two parameters per quantile and the number of parameters to be estimated may become quite large. Moreover, QR not always improves from assuming homoscedasticity: this depends on the actual distribution of the errors. Figure $3 \mathrm{a}$ and $\mathrm{b}$ shows two situations in which the use of the linear QR leads to very different results. Figure $3 \mathrm{a}$ is an optimal situation for using linear QR because the variation of error variance is linearly decreasing with the magnitude of the forecasts and the resulting quantiles well represent the real distribution of the data. On the contrary, in Fig. $3 \mathrm{~b}$ it is not possible to identify a linear variation of the error variance and the use of the linear QR does not provide improved assessments of PU, particularly for high forecast values.

\section{Model conditional processor}

The Model Conditional Processor (MCP) is a Bayesian methodology, proposed by Todini (2008), for estimating the predictive uncertainty. The derivation of the predictive distribution is essentially based on the estimation of a joint predictand-prediction distribution, computed by taking advantage of the model behaviour knowledge acquired through the available historical series. Since the multivariate distributions can be formulated and effectively analytically treated in a very limited number of cases, Krzysztofowicz (1999) suggested transforming the observations and model forecasts in a Gaussian or Normal space via a non parametric transformation, the NQT (Van der Waerden, 1952, 1953a,b). The original variables $y$ and $\widehat{y}$, whose empirical cumulative distribution function has been computed using the Weibull plotting position, are so converted to their transformed values $\eta$ and $\widehat{\eta}$ respectively, following a Normal Standard Distribution. The Weibull plotting position refers to the expected value of the probability of the $i_{\text {th }}$ element of a ranked (smallest to largest) sample of size $n$, which is $\frac{i}{(n+1)}$. Its use is recommended when the form of the distribution is unknown and when unbiased exceedance probabilities are desired. 


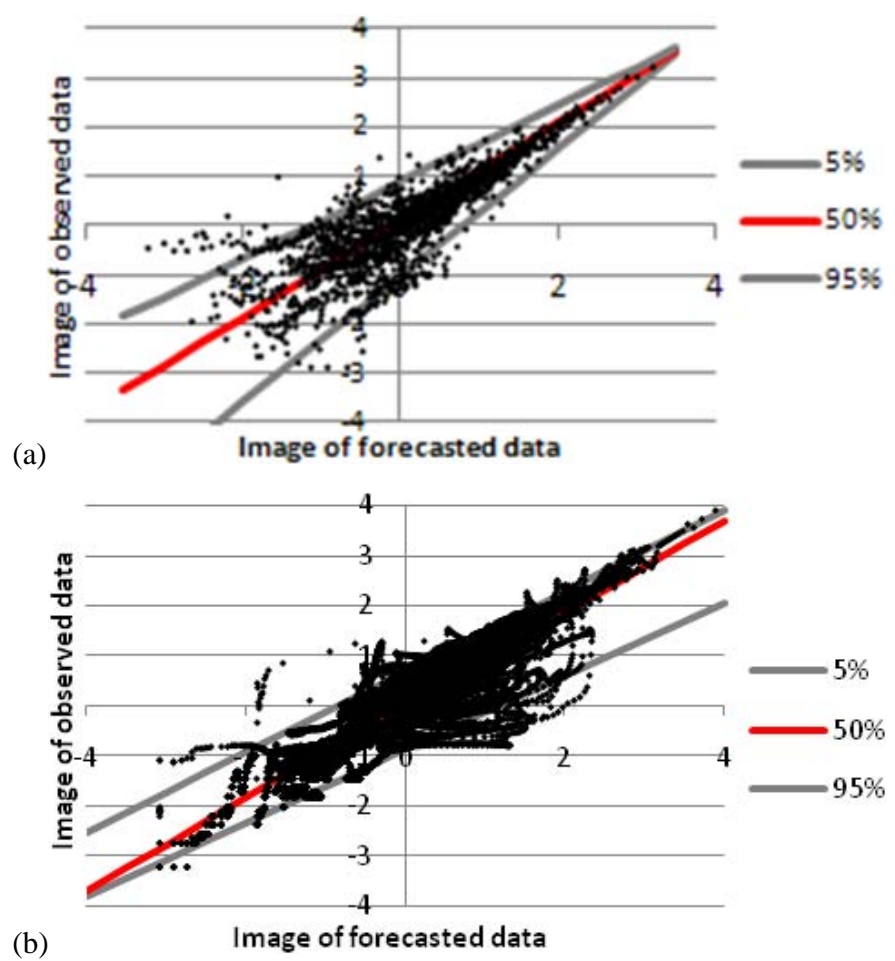

Fig. 3. (a) An optimal situation for using the QR. (b) Poor results are obtained using QR in the situation represented here, which, by the way, is quite common in hydrological applications.

In the Normal space the joint distribution of $\eta$ and $\widehat{\eta}$ can be assumed as a Normal Bivariate, $f(\eta, \widehat{\eta})$, allowing the predictive distribution to be easily computed according to the Bayes theorem, as described in (Todini, 2008). The moments of the predictive distribution in the Normal space are:

$\mu_{\eta \mid \widehat{\eta}}=\rho_{\eta \widehat{\eta}} \cdot \widehat{\eta}$

$\sigma_{\eta \mid \widehat{\eta}}^{2}=1-\rho_{\eta \widehat{\eta}}^{2}$

Therefore, after obtaining the conditional probability in the normal space, the results have to be converted into the real world in order to compute the predictive probability $f(y \mid \widehat{y})$. To do so the predictive density has to be sampled in the Normal space and then the obtained quantiles have to be reconverted into the real space by a reverse process. This is due to the fact that the transformation is highly non linear, and, for instance, the mean value in the Normal space does not correspond to the mean value in the real world, in fact it corresponds to the median (50\% probability) (Todini, 2009). In this process the use of the Weibull plotting position implies the need of using an additional model to fit to the tails of all the variables, namely the observations and the model forecast, in the real space, in order to accommodate probability quantiles larger than $\frac{n}{n+1}$ or lower than $\frac{1}{n+1}$. The choice of the best tail model depends on the actual distribution of the data, in most applications of the MCP the following models have been used, respectively for the lower and the upper tail:

$$
\begin{aligned}
& p(y)=p_{\text {low }} \cdot\left[\frac{y}{y\left(p_{\text {low }}\right)}\right]^{a} \\
& p(y)=1-\left(1-p_{\text {up }}\right) \cdot\left[\frac{y_{\text {max }}-y}{y_{\text {max }}-y\left(p_{\text {up }}\right)}\right]^{b}
\end{aligned}
$$

where $p_{\text {low }}$ and $p_{\text {up }}$ are the lower and upper limits defining the probability values for which the tails will be used; $y\left(p_{\text {low }}\right)$ and $y\left(p_{\text {up }}\right)$ are the values of the variable $y$ correspondent to the probability limits; $y_{\max }$ is the maximum value for which the probability is assumed to be equal to 1 and, although it can be derived through an extreme value analysis, for the sake of simplicity, in the proposed case studies it was assumed to be equal to twice the maximum value ever observed; $a$ and $b$ are the parameters to be estimated. Concerning the lower tail it is assumed that the null probability is assigned to the null value of the variable $y$, that is true when dealing with discharges, but not if $y$ represents level values. In this case it is necessary to refer all the values to the bedstream level, so that the null level is the lowest level possible. Moreover, using level values also $y_{\max }$ must be computed as the double of the maximum level observed referred to the bedstream level.

\subsection{The multi-model case}

The previously described MCP methodology has generated the idea of generalizing the procedure using a multi-normal approach (Todini, 2008). Often, a real time forecasting system is composed by more than one model, or a chain of models, and the emergency manager has to take a decision on the basis of multiple forecasts of the same quantity that may also be very different from each other. It is very difficult to find an objective way to state that one model is better than another, or to assign a correct weight to each forecast in order to extrapolate from all the available information a stochastic forecast that allows the emergency to be managed in the best way.

In order to combine several model forecasts, the MCP can be improved by generalizing the bivariate normal approach to a multivariate normal approach (Mardia et al., 1979). In this case the Multivariate space is composed by $M+1$ variables, that are the observed discharges (or water levels) $y$ and the $M$ predictions $\hat{y}_{k}, k=1, \ldots, M$. Using the NQT, all the variables are converted to their transformed values, $\eta$ and $\hat{\eta}_{k}, k=1, \ldots, M$, in the multi-normal space.

All the variables in the normal space have a standard normal distribution and the predictive uncertainty, defined now as the distribution of the future event conditioned on the forecasts of the $M$ models, can be expressed as $\left(y \mid \hat{y}_{1}, \ldots, \hat{y}_{M}\right)$, for simplicity abbreviated to $f\left(y \mid \hat{\boldsymbol{y}}_{\boldsymbol{k}}\right)$ for the original variable and $f\left(\eta \mid \hat{\boldsymbol{\eta}}_{k}\right)$ in the normal space. 
The joint distribution is a multi-normal distribution with mean and variance

$\boldsymbol{\mu}_{\eta, \hat{\eta}_{k}}=\left[\begin{array}{c}0 \\ \vdots \\ 0\end{array}\right]$

$\Sigma_{\eta, \hat{\eta}_{\mathbf{k}}}=\left[\begin{array}{ccccc}1 & \rho_{\eta \hat{\eta}_{1}} & \rho_{\eta \hat{\eta}_{2}} & \cdots & \rho_{\eta \hat{\eta}_{M}} \\ \rho_{\hat{\eta}_{1} \eta} & 1 & \rho_{\hat{\eta}_{1} \hat{\eta}_{2}} & \ddots & \rho_{\hat{\eta}_{1} \hat{\eta}_{M}} \\ \rho_{\hat{\eta}_{2} \eta} & \rho_{\hat{\eta}_{2} \hat{\eta}_{1}} & \ddots & \ddots & \vdots \\ \vdots & \ddots & \ddots & \ddots & \rho_{\hat{\eta}_{M-1} \hat{\eta}_{M}} \\ \rho_{\hat{\eta}_{M} \eta} & \rho_{\hat{\eta}_{M} \hat{\eta}_{1}} & \cdots & \rho_{\hat{\eta}_{M} \hat{\eta}_{M-1}} & 1\end{array}\right]$

Defining

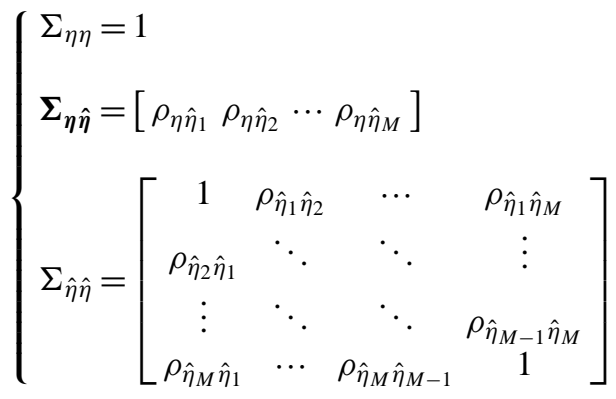

and substituting Eq. (10) in Eq. (9), the cross correlation matrix can also be written as

$\Sigma_{\eta, \hat{\eta}_{\mathbf{k}}}=\left[\begin{array}{cc}\Sigma_{\eta \eta} & \Sigma_{\eta \hat{\eta}} \\ \Sigma_{\eta \hat{\eta}} T & \Sigma_{\hat{\eta} \hat{\eta}}\end{array}\right]$

Then the predictive uncertainty can be expressed as

$f\left(\eta \mid \hat{\boldsymbol{\eta}}_{\boldsymbol{k}}\right)=\frac{f\left(\eta, \hat{\eta}_{1}, \ldots, \hat{\eta}_{M}\right)}{f\left(\hat{\eta}_{1}, \ldots, \hat{\eta}_{M}\right)}$

The solution of Eq. (12) is easily obtained and leads to a normal distribution with moments derived from Eq. (11) as

$\mu_{\eta \mid \hat{\eta}_{k}}=\Sigma_{\eta \hat{\eta}} \cdot \Sigma_{\hat{\eta} \hat{\eta}}-1 \cdot\left[\begin{array}{c}\hat{\eta}_{1} \\ \vdots \\ \hat{\eta}_{M}\end{array}\right]$

$\sigma_{\eta \mid \hat{\eta}_{k}}^{2}=1-\Sigma_{\eta \hat{\eta}} \cdot \Sigma_{\hat{\eta} \hat{\eta}}^{-1} \cdot \Sigma_{\eta \hat{\eta}}^{T}$

Please note that Eq. (13) does not differ from the classical multiple regression results.

As done for the univariate case, the predictive uncertainty in the real world, $f\left(y \mid \hat{\boldsymbol{y}}_{\boldsymbol{k}}\right)$, is obtained by converting $f\left(\eta \mid \hat{\boldsymbol{\eta}}_{\boldsymbol{k}}\right)$ by means of the inverse NQT.

\subsection{Truncated normal joint distributions to account for the error heteroscedasticity}

As mentioned in Sect. 2.3, the assumption of homoscedasticity of the error variance leads to a lack of accuracy in representing the PU, especially at reproducing high flows, because

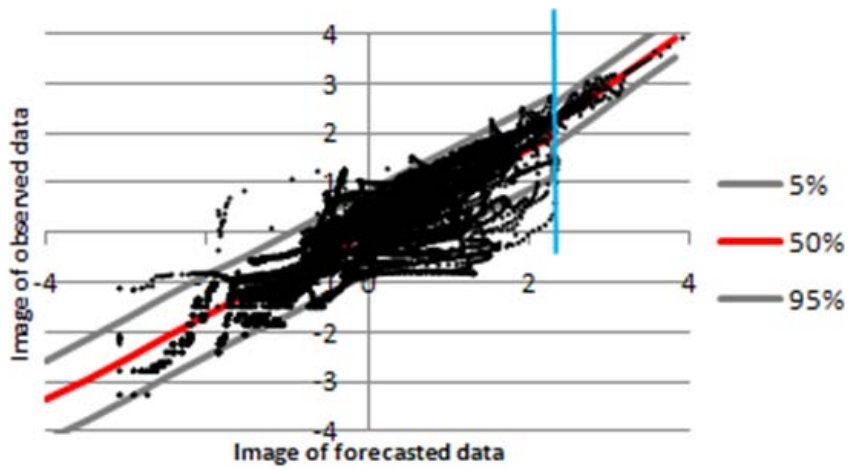

Fig. 4. Truncated normal joint distributions. The division of the Joint Distribution in the normal space into two bivariate truncated normal distributions is shown. The red line represents the modal value, while the grey lines represent the $5 \%$ and the $95 \%$ quantiles. The light blue line represents the threshold used in order to identify the two TNDs.

the NQT tends to increase the variance of the lower values. Moreover, the number of observed and predicted low and medium flows is much larger than that of high flows with the consequence of a higher weight in the determination of the regression or the correlation coefficients used by the different approaches. As a consequence the estimation of high flows in the Normal Space will be affected by a distortion in the mean as well as an overestimation of the variance, which will inevitably increase when returning into the real space. To face this problem an alternative approach has been introduced in the MCP formulation. Namely, within the MCP framework the entire Normal domain is divided into two (or more) subdomains where Truncated Normal Distributions (TNDs) can be used. In this case, the MCP can be applied assuming that the joint distribution in the Normal Space is not unique, but can be divided into two (or more) TNDs. A threshold separating low flows from high flows, in the forecast domain, is relatively easy to be identified. Figure 4 shows the two TNDs that can be used in that case.

The identification of the two TNDs is not immediate, but can be obtained by the following procedure that depends on the number of available forecasting models.

\subsubsection{TNDs with only one forecasting model}

After converting the original variables $y$ and $\widehat{y}$ to their transformed values $\eta$ and $\hat{\eta}$, a threshold $a$ is chosen among the values of $\hat{\eta}$ in order to split the data in two samples, which are assumed to belong to two unknown normal distributions truncated over $\hat{\eta}$ by $a$. If more than one threshold are identified, it is possible to consider more than two TNDs, but in order to avoid the processor to excessively adapt to the calibration data, with the risk of losing generalization ability, it is advisable to use as few TNDs as possible. Usually two TNDs are enough to well represent the distribution of the data in the Normal Space. 
The threshold $a$ can be identified as the value of $\hat{\eta}$ that minimizes the predictive variance of the upper sample (the one representing the high flows) and its search must be lower and upper limited in order to count with significant samples for computing the moments of the truncated distributions. In fact, the moments of these truncated distributions must be estimated by equating them to the sampling moments, as described below.

Taking into account only the sample that includes the high flows, the Truncated Normal distribution for $\hat{\eta}>a$ is

$f(\hat{\eta} \mid \hat{\eta}>a)=\frac{f(\hat{\eta})}{\int_{a}^{+\infty} f(\hat{\eta}) d \hat{\eta}}=\frac{f(\hat{\eta})}{1-F_{\hat{\eta}}(a)}$

with $f(\hat{\eta})$ defined as

$f(\hat{\eta})=\frac{1}{\sqrt{2 \pi} s_{\hat{\eta}}} \exp \left\{-\frac{1}{2}\left(\frac{\hat{\eta}-m_{\hat{\eta}}}{s_{\hat{\eta}}}\right)^{2}\right\}$

where $\hat{m}$ and $\hat{s}$ are the mean and the standard deviation of the non truncated, albeit unknown distribution.

Therefore, the joint distribution is the following truncated normal bivariate distribution

$f(\eta, \hat{\eta} \mid \hat{\eta}>a)=\frac{f(\eta, \hat{\eta})}{\int_{-\infty}^{+\infty}\left[\int_{a}^{+\infty} f(\eta, \hat{\eta}) d \hat{\eta}\right] d \eta}=\frac{f(\eta, \hat{\eta})}{1-F_{\hat{\eta}}(a)}(16)$

Where $f(\eta, \hat{\eta})$ is defined as

$f(\eta, \hat{\eta})=\frac{\exp \left\{-\frac{1}{2}\left[\eta-m_{\eta} \hat{\eta}-m_{\hat{\eta}}\right] S^{-1}\left[\begin{array}{l}\eta-m_{\eta} \\ \hat{\eta}-m_{\hat{\eta}}\end{array}\right]\right\}}{\sqrt{2 \pi|S|}}$

where $\mathbf{S}=\left[\begin{array}{cc}s_{\eta}^{2} & s_{\eta \hat{\eta}} \\ s_{\eta \hat{\eta}} & s_{\hat{\eta}}^{2}\end{array}\right]$.

In Eqs. (15) and (17), the values of $m_{\hat{\eta}}, s_{\hat{\eta}}, m_{\eta}, s_{\eta}$ and $s_{\eta \hat{\eta}}$ are unknown but can be derived from the sampling moments. Applying the Bayes theorem to the TNDs, the predictive uncertainty becomes:

$f(\eta \mid \hat{\eta}>a)=\frac{f(\eta, \hat{\eta} \mid \hat{\eta}>a)}{f(\hat{\eta} \mid \hat{\eta}>a)}=\frac{f(\eta, \hat{\eta})}{f(\hat{\eta})}$

It is normally distributed and its mean and variance are functional on the realization of $\hat{\eta}, \hat{\eta}^{*}>a$

$$
\begin{aligned}
& \mu_{\eta \mid \hat{\eta}=\hat{\eta}^{*}, \hat{\eta}^{*}>a}=m_{\eta}+\frac{s_{\eta \hat{\eta}}}{s_{\hat{\eta}}{ }^{2}}\left(\hat{\eta}^{*}-m_{\hat{\eta}}\right) \\
& \sigma_{\eta \mid \hat{\eta}=\hat{\eta}^{*}, \hat{\eta}^{*}>a}^{2}=s_{\eta}^{2}-\frac{s_{\eta \hat{\eta}}^{2}}{s_{\hat{\eta}}^{2}}
\end{aligned}
$$

According to the truncated multi-normal distribution theory (Tallis, 1961), the previous equations allow the PU to be defined in the Normal Space as a Normal Distribution with mean and variance:

$$
\begin{aligned}
& \mu_{\eta \mid \hat{\eta}=\hat{\eta}^{*}, \widehat{\eta}^{*}>a}=\mu_{\eta}+\frac{\sigma_{\eta \hat{\eta}}}{\sigma_{\hat{\eta}}^{2}}\left(\hat{\eta}^{*}-\mu_{\hat{\eta}}\right) \\
& \sigma_{\eta \mid \hat{\eta}=\widehat{\eta}^{*}, \widehat{\eta}^{*}>a}^{2}=\sigma_{\eta}^{2}-\frac{\sigma_{\eta \hat{\eta}^{2}}}{\sigma_{\hat{\eta}^{2}}^{2}}
\end{aligned}
$$

Here $\mu_{\eta}, \mu_{\hat{\eta}}$ and $\sigma_{\eta}, \sigma_{\hat{\eta}}$ are respectively the sample means and standard deviations of $\eta \mid \hat{\eta}>a$ and $\hat{\eta} \mid \hat{\eta}>a$. These moments are obviously computed considering only the data included in the upper sample.

Considering now the lower sample and a relaziation of $\hat{\eta}$, $\hat{\eta}^{*}<a$, Eqs. (14) and (16) become, respectively

$$
\begin{aligned}
& f(\hat{\eta} \mid \hat{\eta}<a)=\frac{f(\hat{\eta})}{\int_{-\infty}^{a} f(\hat{\eta}) d \hat{\eta}}=\frac{f(\hat{\eta})}{F_{\hat{\eta}}(a)} \\
& f(\eta, \hat{\eta} \mid \hat{\eta}<a)=\frac{f(\eta, \hat{\eta})}{\int_{-\infty}^{+\infty}\left[\int_{-\infty}^{a} f(\eta, \hat{\eta}) d \hat{\eta}\right] d \eta}=\frac{f(\eta, \hat{\eta})}{F_{\hat{\eta}}(a)}
\end{aligned}
$$

If the same procedure carried out for the upper sample is applied to the lower sample, the predictive uncertainty is obtained with the following equation

$$
\begin{aligned}
& \mu_{\eta \mid \hat{\eta}=\hat{\eta}^{*}, \hat{\eta}^{*}<a}=\mu_{\eta}+\frac{\sigma_{\eta \hat{\eta}}}{\sigma_{\hat{\eta}}^{2}}\left(\hat{\eta}^{*}-\mu_{\hat{\eta}}\right) \\
& \sigma_{\eta \mid \hat{\eta}=\hat{\eta}^{*}, \hat{\eta}^{*}<a}^{2}=\sigma_{\eta}^{2}-\frac{\sigma_{\eta \hat{\eta}}^{2}}{\sigma_{\hat{\eta}}^{2}}
\end{aligned}
$$

Please note that Eq. 23 is equal to Eq. 18, but in this case $\mu_{\eta}$, $\mu_{\hat{\eta}}, \sigma_{\eta}$ and $\sigma_{\hat{\eta}}$ are computed taking into account only the data of the lower sample.

\subsubsection{TNDs with more than one forecasting model}

When dealing with more than one model, the procedure becomes a bit more difficult. The threshold should be identified for each model and the joint distribution would be represented by $2^{M}$ Multivariate Truncated Normal Distributions (MTNDs) (where $M$ is the number of models) that include all the possible simultaneous combinations of each model overtopping or not its respective threshold. The moments of each MTNDs should be obtained by means of the sampling moments computation, but unfortunately in real cases often the available data are not enough to identify representative samples and the MTNDs cannot be well assessed.

In order to avoid this situation the problem can be tackled with a different approach. The MCP can be applied in three phases. Firstly, each model is processed separately using the TNDs as described above. In this phase, for each model its threshold is identified. In the second phase, the series of expected values of each model simulation (previously obtained) are combined again using two MTNDs. The split of the multi-variate Normal Space in two parts is obtained identifying the hyperplane that includes the point $\left[\left(\eta=0, \hat{\eta}_{i}=\right.\right.$ $a), \forall i=1 . . M]$ and is perpendicular to the straight line that links the origin to that point. This hyperplane is identified by the following equation:

$\sum_{i=1}^{M} \hat{\eta}_{i}=M \cdot a$ 


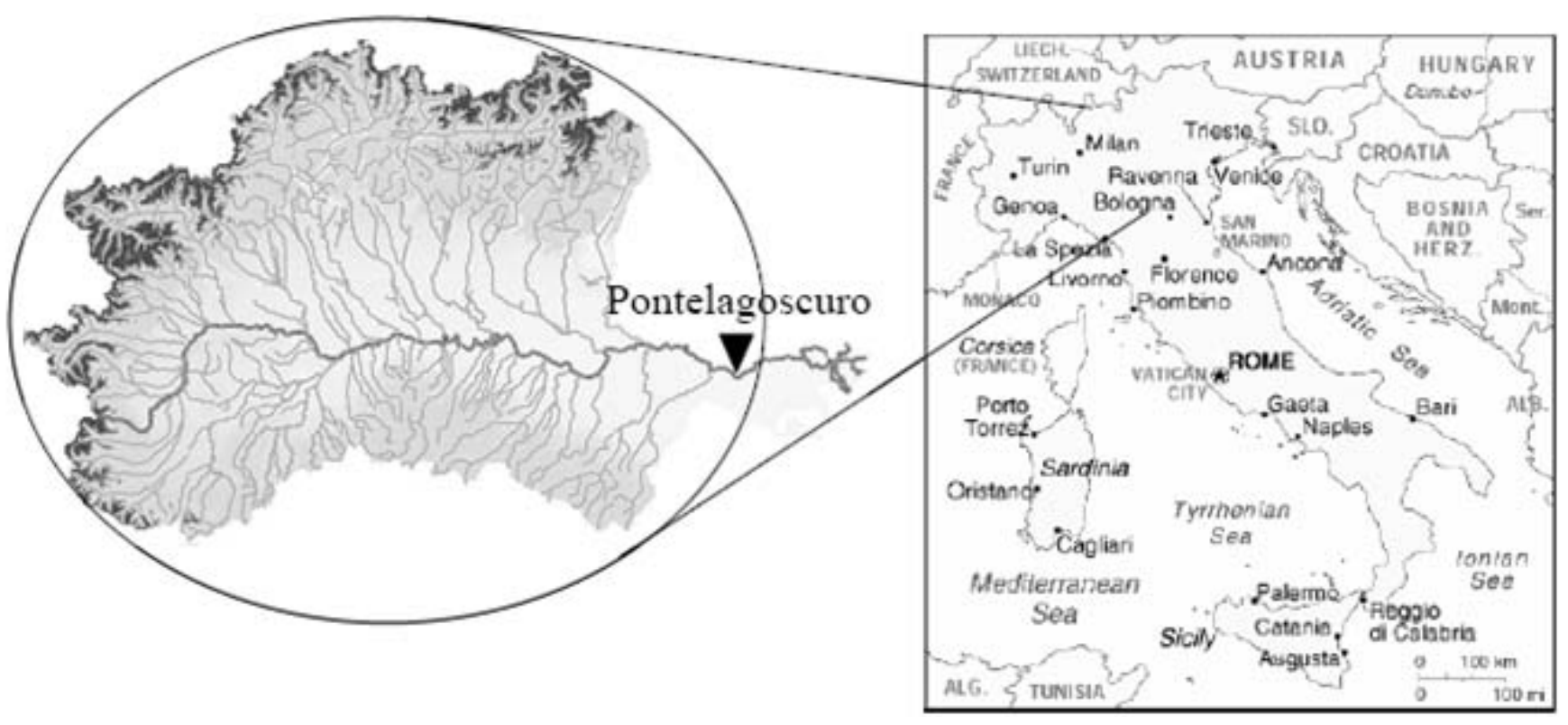

Fig. 5. The River Po catchment in Italy and the location of the gauging station of Pontelagoscuro.

The value of $a$ is again identified as the one that minimizes the predictive variance of the upper sample. Finally, in the third phase the series of expected values computed in the second phase is processed using the TNDs as described in Sect. 3.2.1.

Concerning the second phase, when the value $a$ is identified the data are split in two samples, one containing the data below the truncation hyperplane and the other above it. After computing the sampling moments for each sample, defining $H_{p}=\sum_{i=1}^{M} \hat{\eta}_{i}$ and following the truncated multi-normal distribution theory (Tallis, 1961), it can be demonstrated that the PU in the normal space, for the sample above the truncation hyperplane, is defined as a normal distribution with mean and variance

$$
\begin{aligned}
& \mu_{\eta \mid \hat{\boldsymbol{\eta}}=\hat{\eta}^{*}, H_{p}^{*}>M \cdot a}=\mu+\Sigma_{\eta \hat{\eta}} \cdot \Sigma_{\hat{\eta} \hat{\eta}}{ }^{-1} \cdot\left(\hat{\eta}^{*}-\hat{\boldsymbol{\mu}}\right) \\
& \sigma_{\eta \mid \hat{\eta}=\hat{\eta}^{*}, H_{p}^{*}>M \cdot a}^{2}=\Sigma_{\eta \eta}-\Sigma_{\eta \hat{\eta}} \cdot \Sigma_{\hat{\eta} \hat{\eta}}-1 \cdot \Sigma_{\eta \hat{\eta}}^{T}
\end{aligned}
$$

Here $\mu$ and $\hat{\mu}$ are, respectively the sample means of $\eta \mid H_{p}>M \cdot a$ and $\hat{\eta} \mid H_{p}>M \cdot a$ and $\Sigma_{\eta \eta}, \Sigma_{\eta \hat{\eta}}, \Sigma_{\hat{\eta} \hat{\eta}}$ are the components of the covariance matrix of $\eta, \hat{\eta} \mid H_{p}>M \cdot a$.

Considering now the sample below the truncation hyperplane, the mean and variance of PU in normal space are

$$
\begin{aligned}
& \mu_{\eta \mid \hat{\eta}=\hat{\eta}^{*}, H_{p}^{*}<M \cdot a}=\mu+\Sigma_{\eta \hat{\eta}} \cdot \Sigma_{\hat{\eta} \hat{\eta}}^{-1} \cdot\left(\hat{\eta}^{*}-\hat{\boldsymbol{\mu}}\right) \\
& \sigma_{\eta \mid \hat{\eta}=\hat{\eta}^{*}, H_{p}^{*}<M \cdot a}^{2}=\Sigma_{\eta \eta}-\Sigma_{\eta \hat{\eta}} \cdot \Sigma_{\hat{\eta} \hat{\eta}}^{-1} \cdot \Sigma_{\eta \hat{\eta}}^{T}
\end{aligned}
$$

Please note that Eq. (26) is equal to Eq. (25), but in this case $\mu, \hat{\mu}, \Sigma_{\eta \eta}, \Sigma_{\eta \hat{\eta}}$ and $\Sigma_{\hat{\eta} \hat{\eta}}$ are computed taking into account only the data of the lower sample.

\section{Examples of application}

Two application examples will be shown in this paper in order to illustrate the benefits of using the proposed methodology. The first example is an operational one, where the predictand is the observed water level. It refers to a flood forecasting system on the Po river in Italy and shows that the MCP approach is well justified for both the full or truncated normal approaches. The second example is set up in order to illustrate the benefits of using both the truncated normal approach as well as the multi model approach. In this case, concerning a recent comparison of distributed hydrological models, the discharges were the only available data, while the water level data were not available. Therefore, bearing in mind the observations made in Sect. 1.2 about the predictand to be chosen, the illustration of the MCP approach and the relevant benefits is based on the solely available discharge records.

\subsection{The Po river example}

\subsubsection{Case study and available data}

The River Po is the largest Italian river with length $650 \mathrm{~km}$ and a catchment area of approximately $70000 \mathrm{~km}^{2}$, covering most of Northern Italy (Fig. 5). The river originates in the North-West of Italy, near the border with France at an altitude of $2000 \mathrm{~m}$ and flows Easterly into the Adriatic Sea after crossing four of the most industrialized and populated Italian regions. Flood risks are steadily growing with increasing urbanization, the expansion of inhabited areas close to the river bed and the consequent increased number of people directly affected by severe floods. The need for effective 
real time flood forecasting and emergency management became extremely clear during the 2000 Po flood, as well as in the inundation of Torino in 2001. Currently a flood forecasting system, based on the PAB hydraulic model (Todini and Bossi, 1986) combined with the Kalman Filter based algorithm MISP (Todini, 1978) is operational with forecasting horizons up to $36 \mathrm{~h}$ in advance. There are several river sections where flood forecasts are issued, but the most important one is the ending section of the river prior to its delta where the level gauging station of Pontelagoscuro is located (Fig. 5). Flood forecasting in Pontelagoscuro is an extremely important issue because the river is here characterized by a suspended bed over a flat plain only protected by high earthen dykes, whose failure could cause dramatic consequences.

The data used as predictands in this work are the measured water levels at Pontelagoscuro, which have been automatically collected in real time since 1993 by a network of telemetering gauges, while the predictors are the water level forecasts produced by the operational flood forecasting system corrected by the Kalman Filter. Nine full years of hourly data were used in this experiment, from January 2000 to December 2008, in order to assess the properties of the different uncertainty processors. The complete data set has been divided in two parts, four years to calibrate the MCP and five years to validate it. All the analysis and results presented in the following sections are based on validation data.

\subsubsection{Predictive uncertainty assessment}

In the case of the Po river, the assessment of predictive uncertainty is made for a forecasting horizon of $36 \mathrm{~h}$ at Pontelagoscuro. Although the hydraulic model performances are quite adequate, the use of the MCP processor to provide the expected value of the predictand given the model forecasts, produces a substantial improvement by practically eliminating all the bias and by reducing the standard error (see Fig. 6). On the contrary, in this case the use of the TNDs, instead of the standard ND, produces a rather small reduction of the uncertainty band, due to the fact that both the hypotheses on the linearity of the relation between observed and modeled normal transformed variables, and on the homoschedasticity of errors, are certainly appropriate, as can be see from Fig. 7, which shows that the spread of the data is rather narrow and more or less constant over the entire field. Nonetheless the use of the TNDs slightly reduces the standard error of high water levels as can be seen in Fig. 8.

\subsubsection{Probability of exceeding an alert threshold assessment}

As can be seen from Fig. 9, a rather effective operational decision supporting tool can be set up by setting the probability threshold at 0.5. Fig. 9 shows an example of $36 \mathrm{~h}$ in advance prediction, during the validation period, when the water level

\section{BIAS [m]}
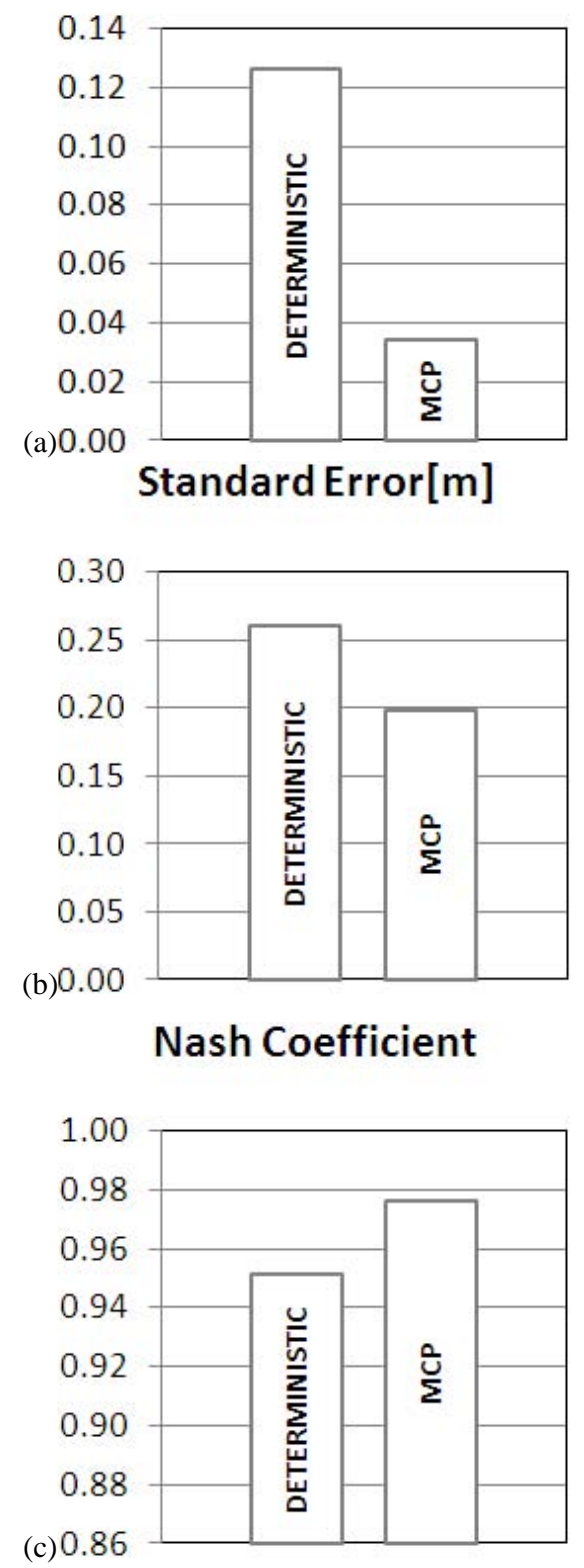

Fig. 6. Comparison between the evaluation indexes for the deterministic model and those obtained from the PU expected value provided by the MCP for the entire validation period. (a) Bias; (b) Standard Error; (c) Nash-Sutcliffe coefficient.

actually overtopped the threshold (a warning threshold in this case). The lower section of Fig. 9 shows the observed values (continuous line), the deterministic forecast (dotted line) and the expected value conditioned to the model forecast (dashed line), as well as the $90 \%$ uncertainty band (grey area) and the alarm threshold set to $0 \mathrm{~m}$ (horizontal dashed line). The upper section represents the probability of exceeding the alarm threshold, the observed binary response (continuous line) and 


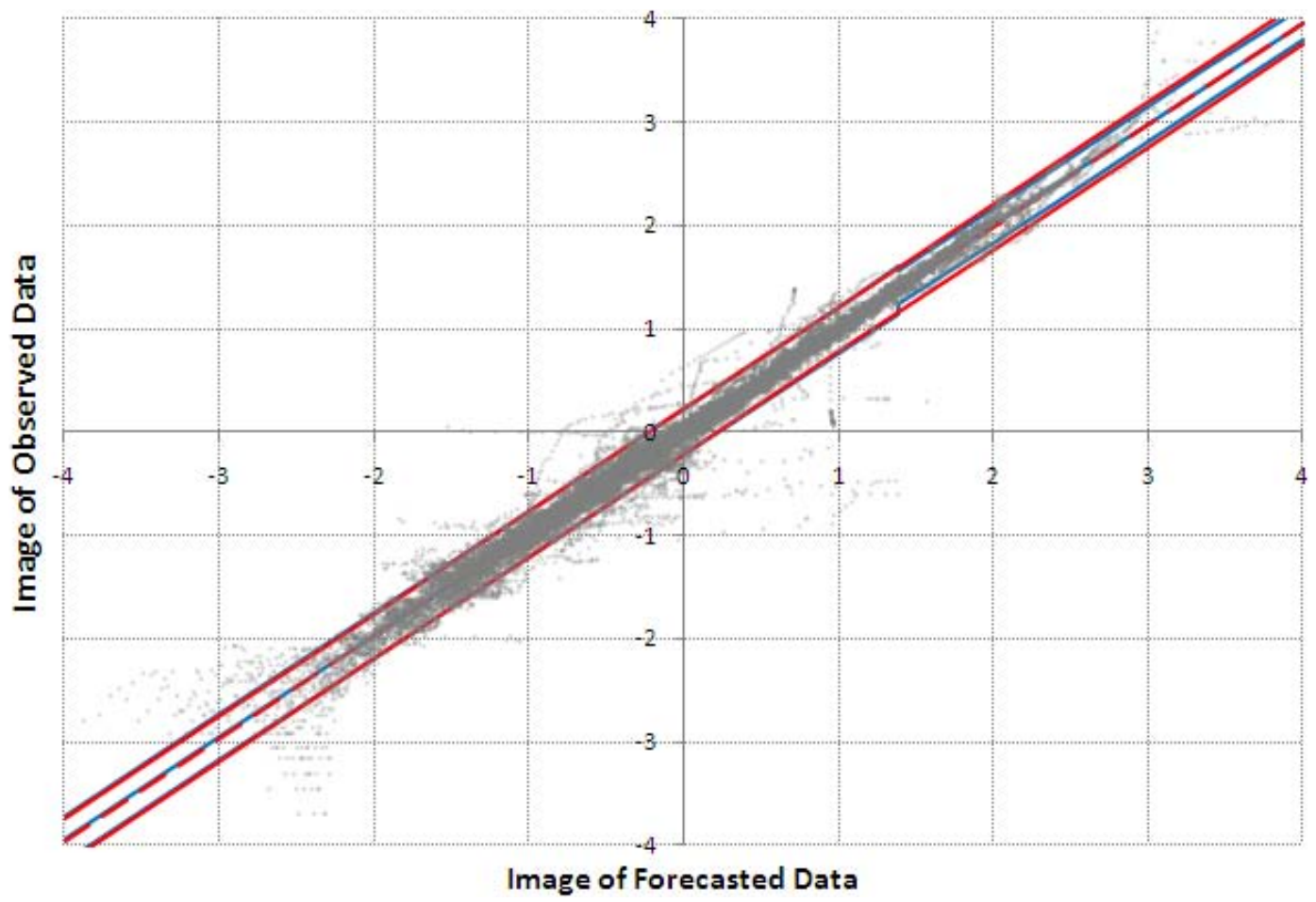

Fig. 7. Representation of the Normal Space obtained applying the MCP to the Po river. The full red lines represent the $5 \%$ and $95 \%$ quantiles and the dashed red line the $50 \%$ quantile obtained without using the TNDs methodology. The blue lines represent the quantiles obtained using the TNDs methodology.

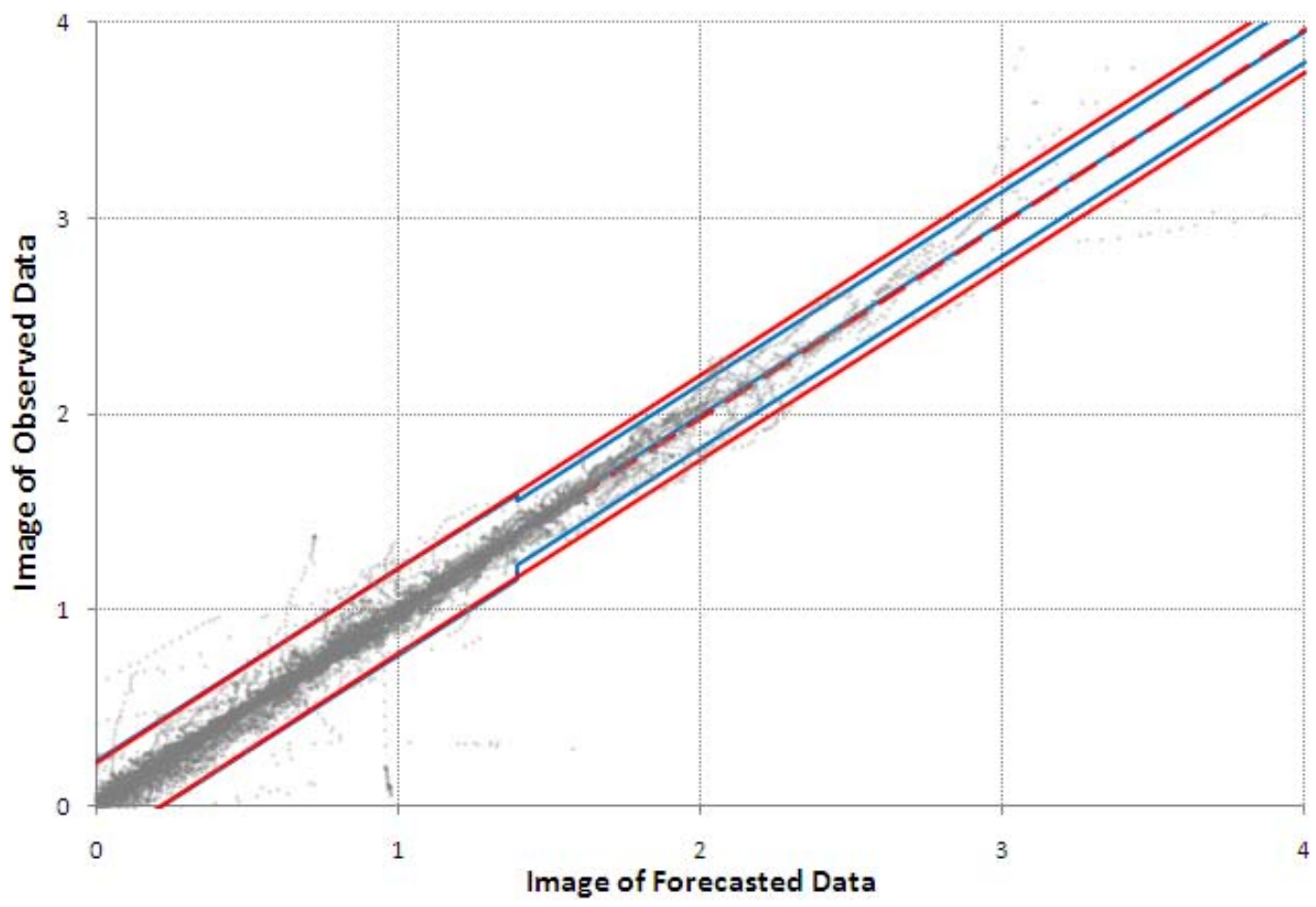

Fig. 8. Zoom of the high values shown in Fig. 7. The full red lines represent the $5 \%$ and $95 \%$ quantiles and the dashed red line the $50 \%$ quantile obtained without using the TNDs methodology. The blue lines represent the quantiles obtained using the TNDs methodology. 


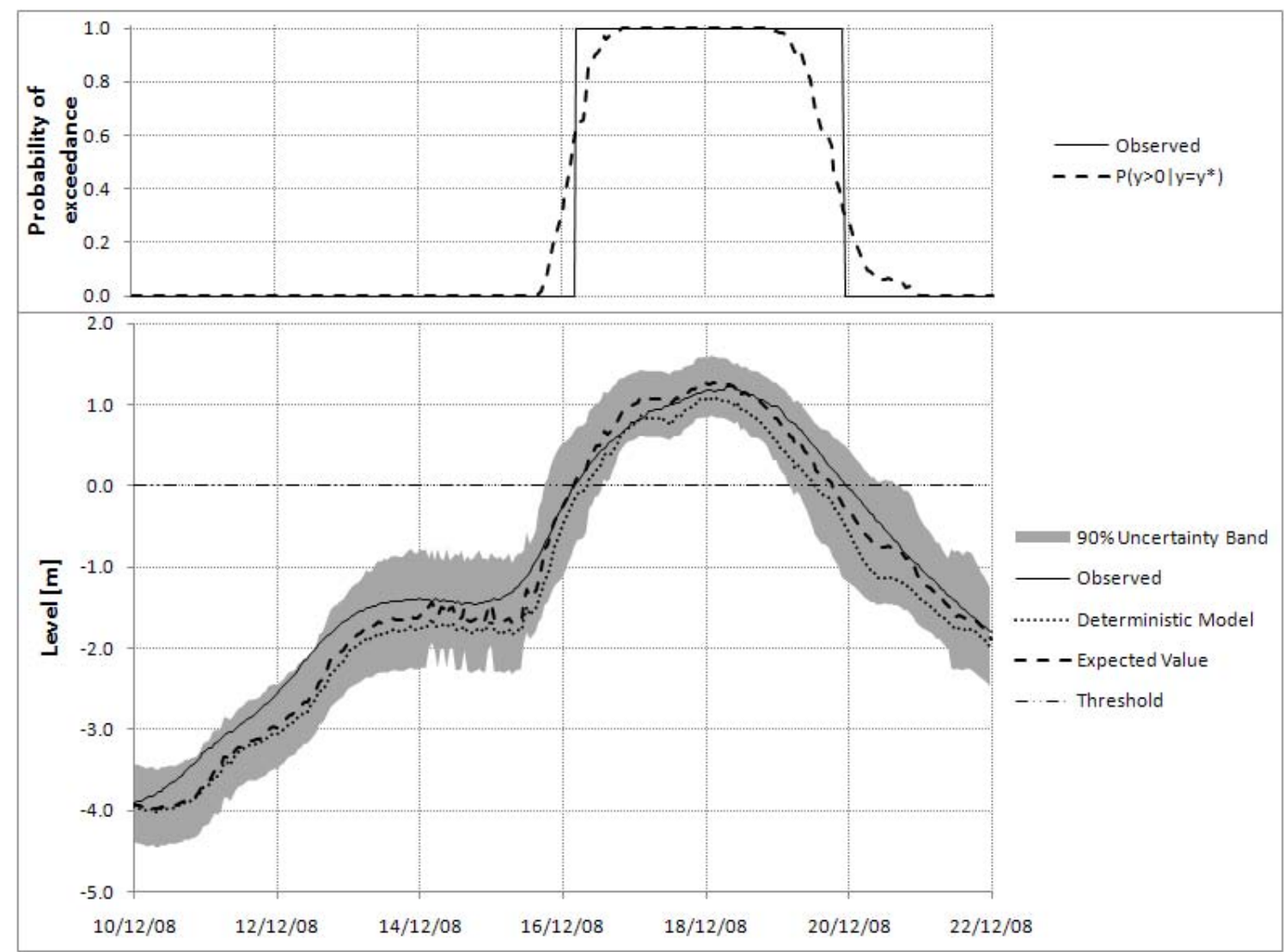

Fig. 9. Flood event for the validation period predicted $36 \mathrm{~h}$ in advance. The lower panel represents the level forecast; observed values (continuous line); deterministic forecast (dotted line); expected value conditioned to the model forecast (dashed line); $90 \%$ uncertainty band (grey area); and alarm threshold of $0 \mathrm{~m}$ (horizontal dashed line). The upper panel represents the probability of exceeding the alarm threshold; observed binary response (continuous line) and probability of exceeding the threshold computed by the MCP (dashed line).

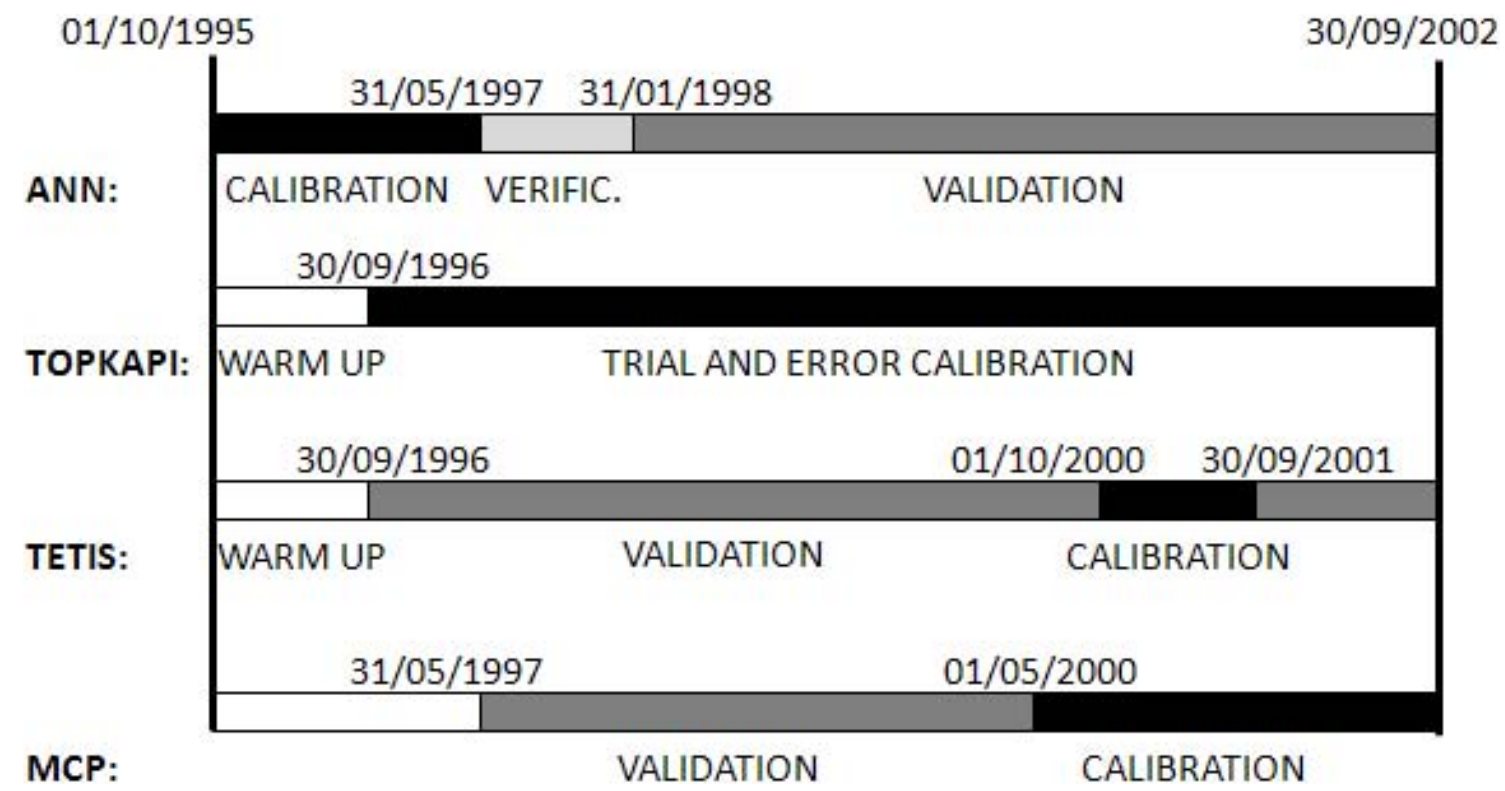

Fig. 10. Schematization of the available data division for calibrating and validating the models and the MCP. 


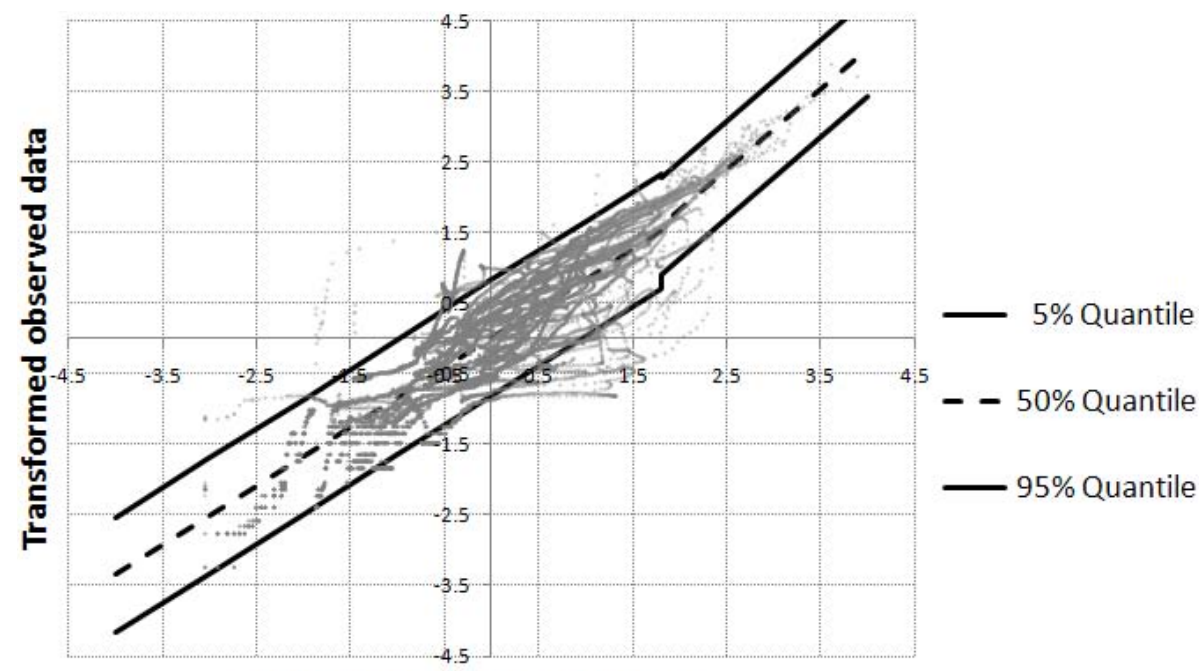

(a)

Transformed forecasted data

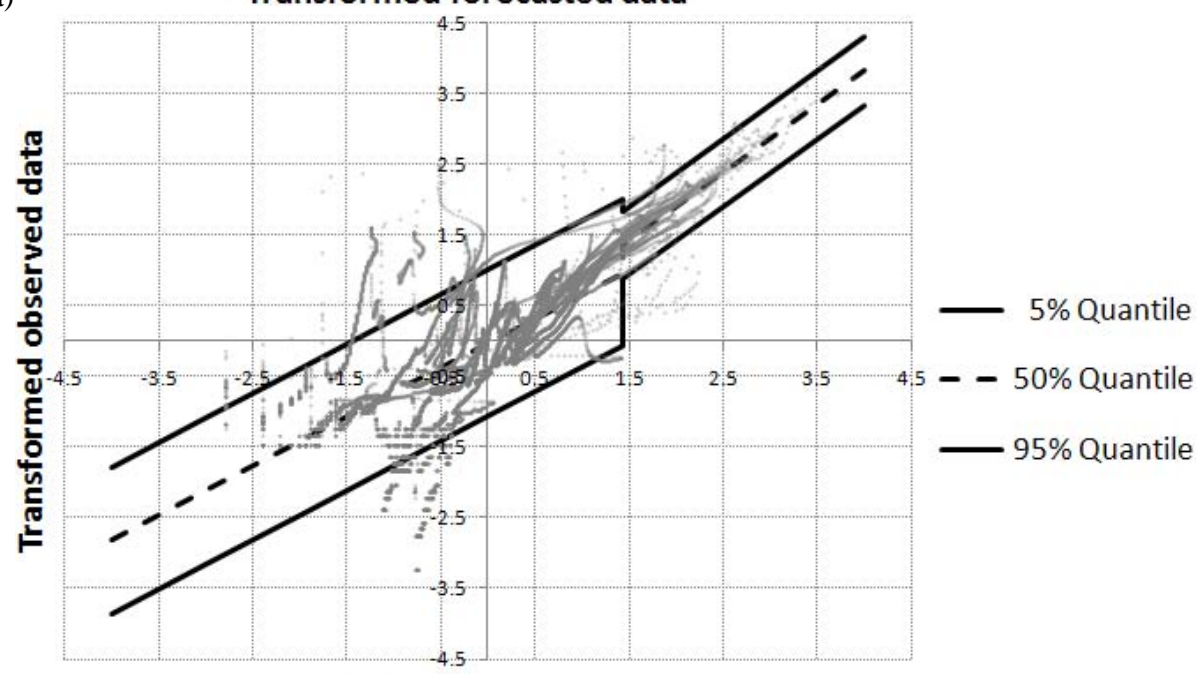

(b)

\section{Transformed forecasted data}

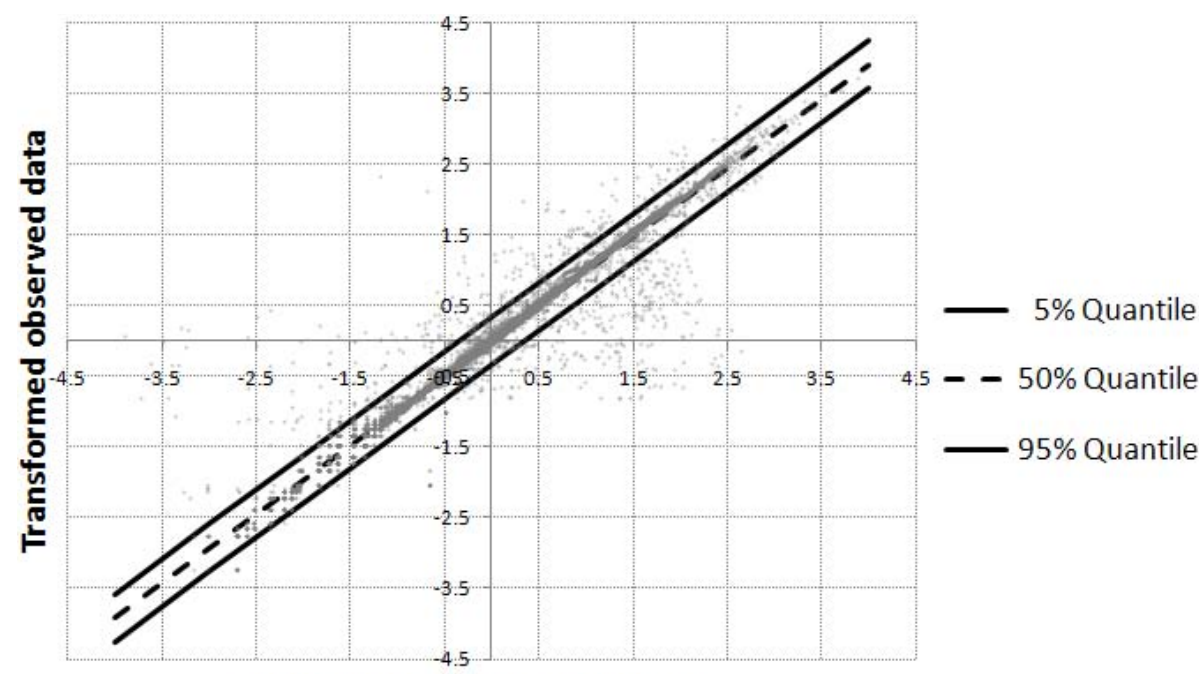

(c)

\section{Transformed forecasted data}

Fig. 11. Representation of the Normal Space obtained using the MCP with the TOPKAPI (a), TETIS (b) and ANN (c) forecasts. 
(a)
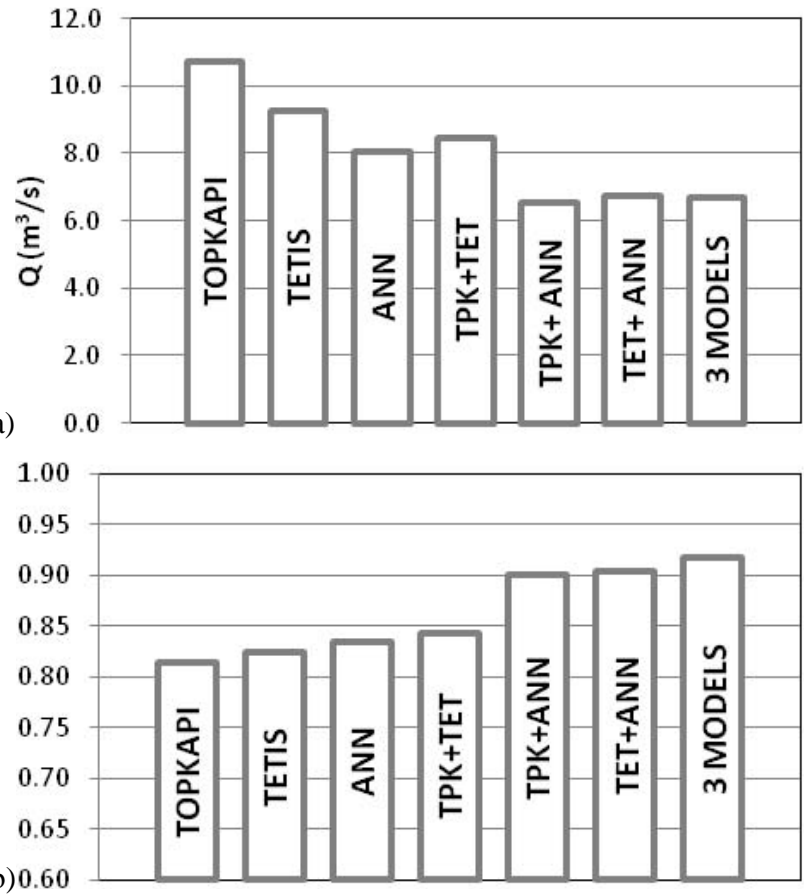

Fig. 12. Evaluation indexes for TOPKAPIi model (TPK), TETIS model (TET), ANN model and their combinations during the entire validation period of the MCP. (a) Standard Error; (b) Nash-Sutcliffe coefficient.

the probability of exceeding the threshold computed using the MCP approach (dashed line). It can be seen that all the forecasts are quite adequate in this example while the probability of exceeding the threshold takes values larger than 0.5 , closely matching the observed binary response.

\subsection{The Baron Fork river example}

\subsubsection{Case study and available data}

The NOAA's National Weather Service, has provided a long series of observed discharge and precipitation data for the Baron Fork River, OK (USA) within the frame of the DMIP 2 Project. Using this data set three models were implemented: two physically based hydrological models, the TOPKAPI model (Todini and Ciarapica, 2001; Liu and Todini, 2002) and TETIS model (Francés et al., 2007; Velez et al., 2009), and a data driven model based on Artificial Neural Networks. The catchment has a drainage area of about $800 \mathrm{~km}^{2}$ at the measurement station of Eldon with a concentration time of approximately $10 \mathrm{~h}$ and a mean slope around $0.25 \%$. Some kilometers downstream Eldon the river flows into the Illinois river. The simulations provided by the three models have been processed using the MCP, firstly each model separately and then combining them.

The available meteorological data consisted in hourly rain and temperature grids with a $4 \mathrm{~km}$ resolution between
1 October 1995 and 30 September 2002. During the same period, also the observed discharges at the measurement station of Eldon were available. Concerning the available data, it is worth mentioning that no water level or rating curve observations were available to the participants involved in the DMIP2 Project. For this reason, the discharge has been used as predictand.

\subsubsection{The real time flood forecasting models}

The TOPKAPI model has been developed at the University of Bologna (Todini and Ciarapica, 2001; Liu and Todini, 2002), it is composed of six components that take into account the surface, sub-surface and deep flows, the channel routing, the snow accumulation/melt and the evapotranspiration processes. The application domain is divided in cells where the mass and momentum balance are solved at every time step. The model has been calibrated by a trial and error procedure considering the period between 1 October 1996 and 30 September 2002; the year included between 1 October 1995 and 30 September 1996 has been used as "warm up" period, allowing the model to reach a reasonable initial state.

In the TETIS model, developed by the Polytechnic University of Valencia (Francés et al., 2007; Velez et al., 2009), the conceptual scheme, at each cell, consists of a series of 5 connected tanks, each one of them representing different water storages in the soil column. The vertical connections between tanks describe the precipitation, evapotranspiration, infiltration and percolation processes, whereas, the horizontal flows represent the main hydrological processes as: snowmelt, overland runoff, interflow and base flow. The routing along the channel network couples its geomorphologic characteristics with the kinematic wave approach. The TETIS model has an automatic calibration procedure that has been used to calibrate the model considering the hydrological year included between October 2000 and September 2001. Also for the TETIS model, the first year of data has been used as "warm up" period and with the remaining data the model has been validated.

The Artificial Neural Network model includes two main phases. Firstly the data were divided in three groups by means of a Self Organizing Map (SOM) network that allows the data to be automatically classified. (Kohonen, 1990; Pujol, 2009). If the time at which the prediction is done is called $t_{0}$, the input data to the SOM network are the accumulated precipitation of 2 days before $t_{0}$, the discharge observed at $t_{0}$ and the gradient of the discharge during $2 \mathrm{~h}$ before $t_{0}$. The SOM network has been calibrated using the data included between 1 October 1995 and 31 May 1997, the remaining data until 30 September 2002 have been used for the validation. The three sets of data obtained by the automatic classification have been used separately in order to calibrate three different Multi Layer Perceptron (MLP) networks (Werbos, 1974, 1988, 1990; Parker, 1987; Pujol, 2009), whose input data are the observed precipitation during $13 \mathrm{~h}$ before $t_{0}$ and 


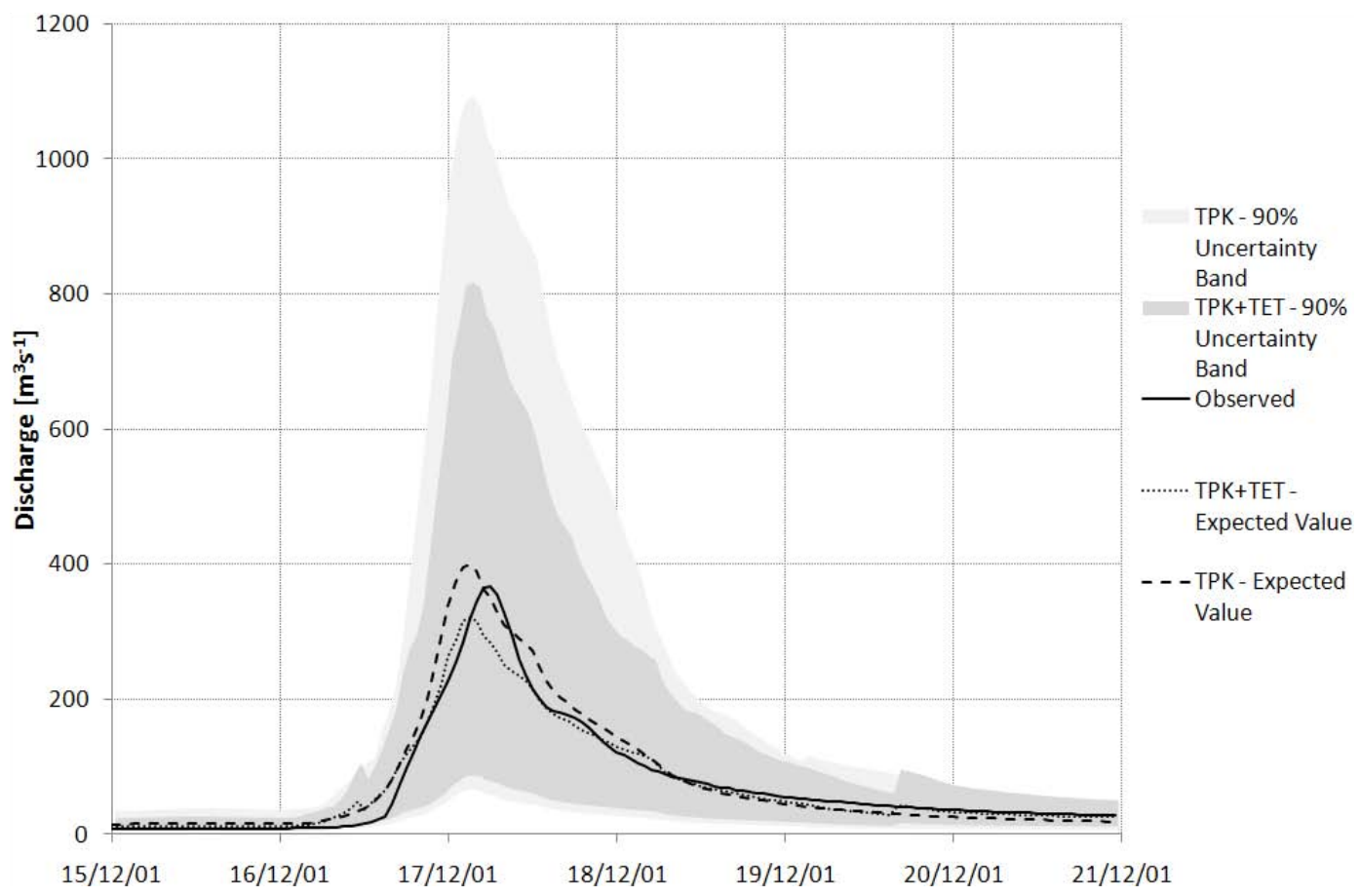

Fig. 13. Comparison between the PU computed with one or two models on a flood event for the calibration period. Observed discharges (black line); expected value conditioned only to the TOPKAPI forecast (dashed line); expected value conditioned to the TOPKAPI and TETIS forecasts (dotted line); $90 \%$ uncertainty band conditioned to the TOPKAPI forecast (light grey band); $90 \%$ uncertainty band conditioned to the TOPKAPI and TETIS forecasts (grey band).

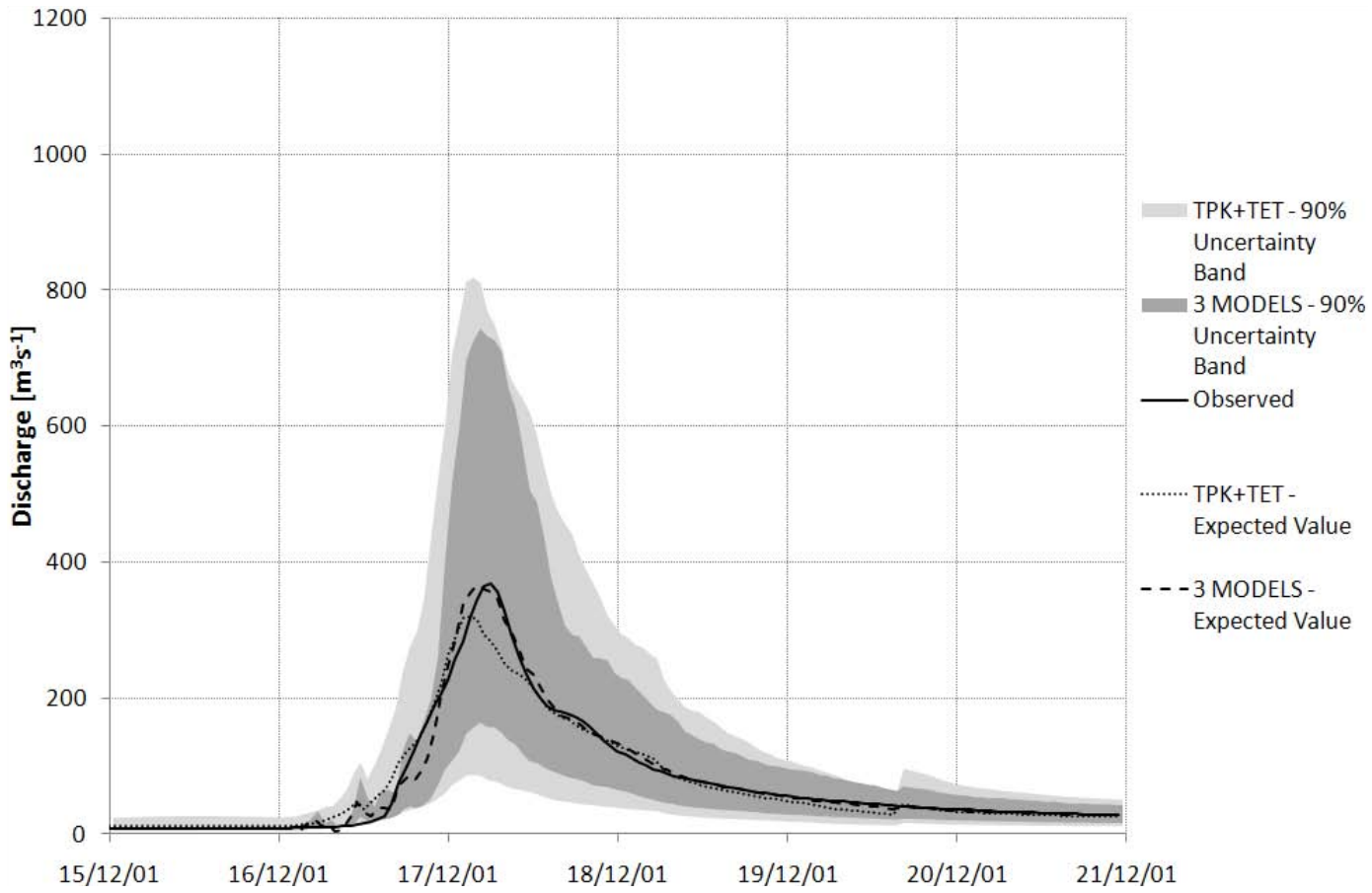

Fig. 14. Comparison between the PU computed combining, two or three models on a flood event for the calibration period. Observed discharges (black line); expected value conditioned only to the TOPKAPI and TETIS forecasts (dotted line); expected value conditioned to the TOPKAPI, TETIS and ANN forecasts (dashed line); $90 \%$ uncertainty band conditioned to the TOPKAPI and TETIS forecasts (light grey band); $90 \%$ uncertainty band conditioned to the TOPKAPI, TETIS and ANN forecasts (grey band). 


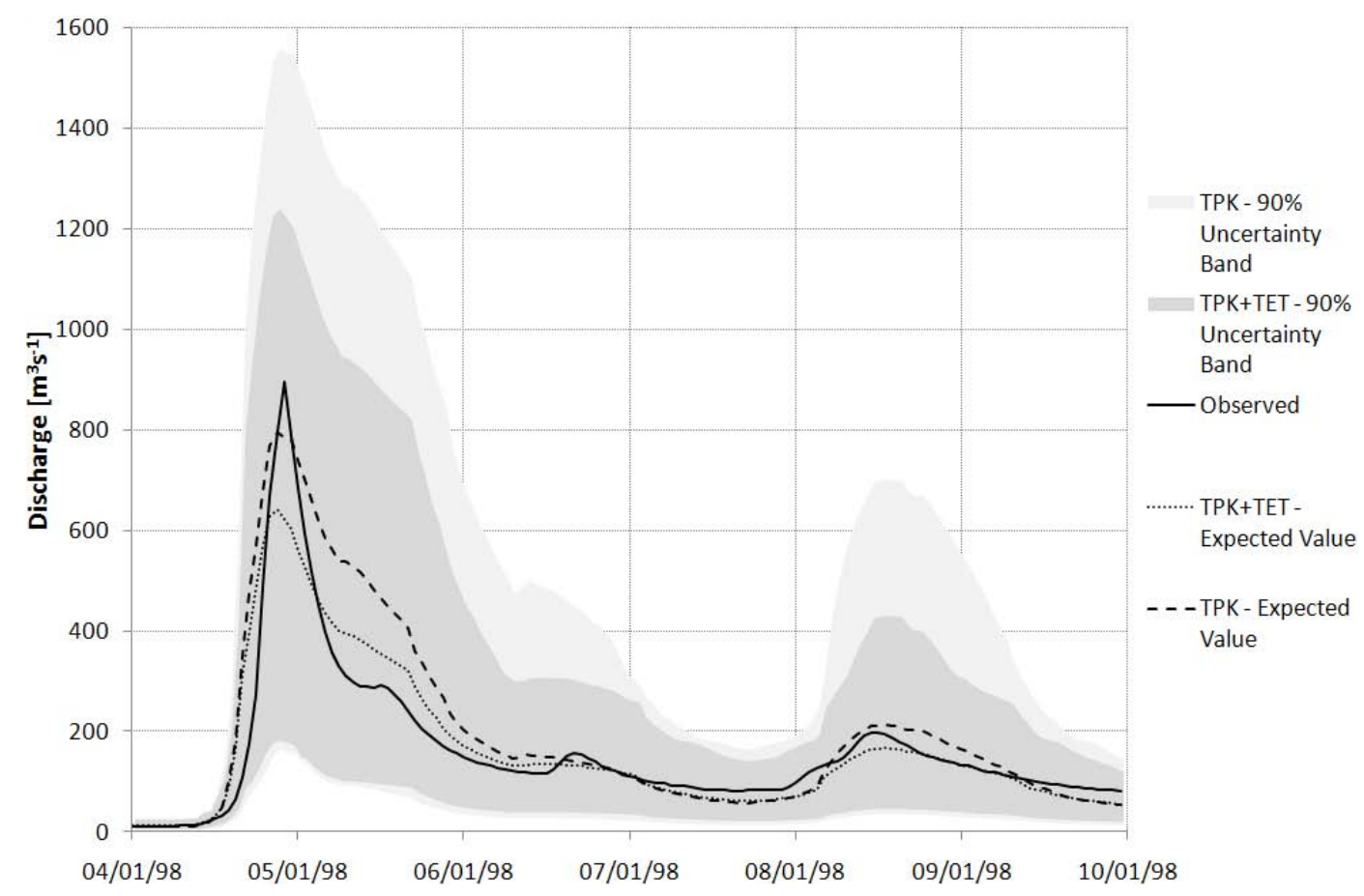

Fig. 15. Comparison between the PU computed with one or two models on a flood event for the validation period. Observed discharges (black line); expected value conditioned only to the TOPKAPI forecast (dashed line); expected value conditioned to the TOPKAPI and TETIS forecasts (dotted line); $90 \%$ uncertainty band conditioned to the TOPKAPI forecast (light grey band); $90 \%$ uncertainty band conditioned to the TOPKAPI and TETIS forecasts (grey band).

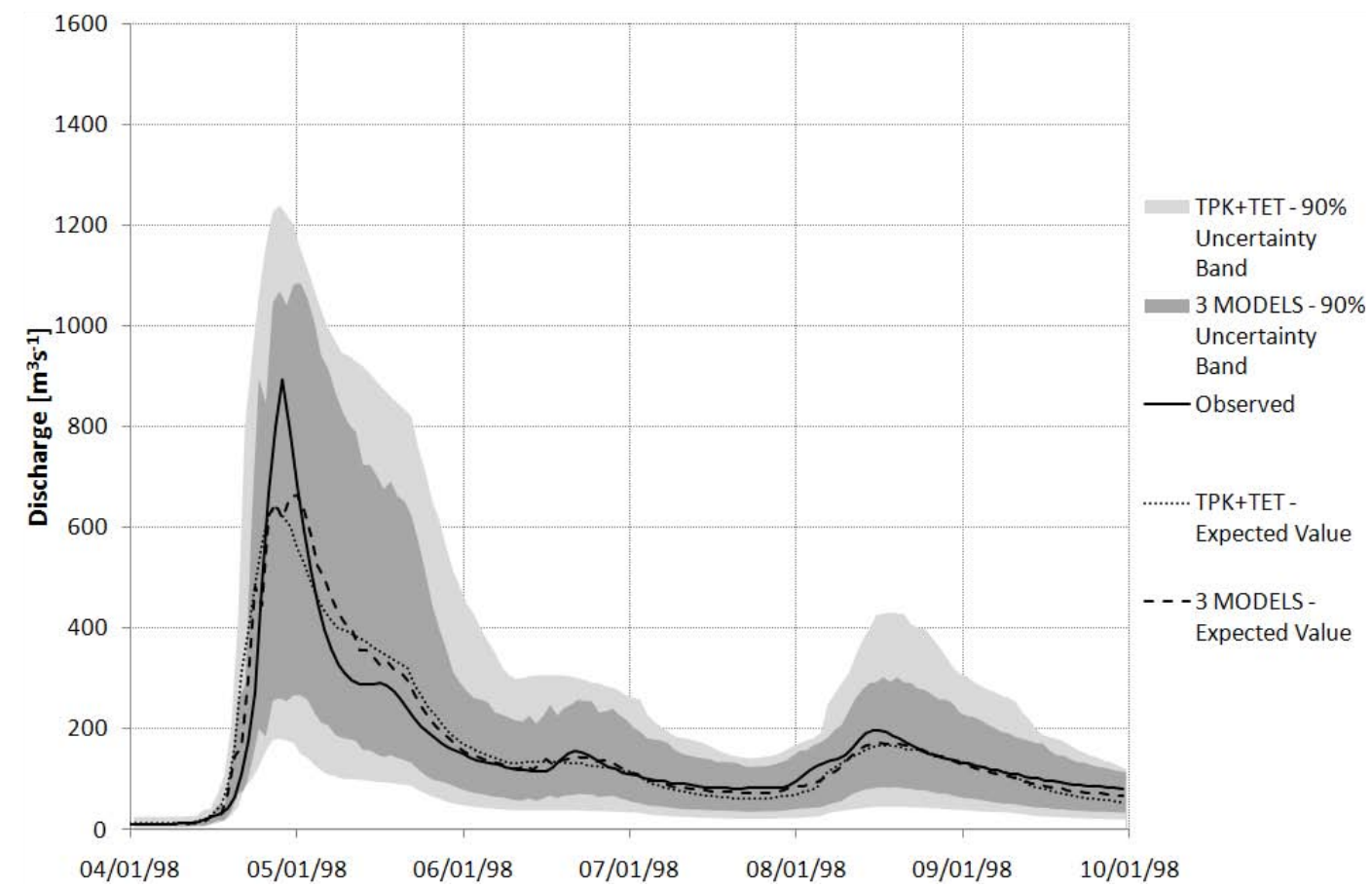

Fig. 16. Comparison between the PU computed combining, two or three models on a flood event for the validation period. Observed discharges (black line); expected value conditioned only to the TOPKAPI and TETIS forecasts (dotted line); expected value conditioned to the TOPKAPI, TETIS and ANN forecasts (dashed line); $90 \%$ uncertainty band conditioned to the TOPKAPI and TETIS forecasts (light grey band); $90 \%$ uncertainty band conditioned to the TOPKAPI, TETIS and ANN forecasts (grey band). 
Table 1. Probability that the true value exceeds the $350 \mathrm{~m}^{3} \mathrm{~s}^{-1}$ threshold when the expected value of prediction equals $250 \mathrm{~m}^{3} \mathrm{~s}^{-1}$, computed for each model and their Bayesian combination.

\begin{tabular}{cccc}
\hline \multicolumn{4}{c}{$P\left(y>350 \mathrm{~m}^{3} \mathrm{~s}^{-1} \mid \hat{y}=250 \mathrm{~m}^{3} \mathrm{~s}^{-1}\right)$} \\
\hline TOPKAPI & TETIS & ANN & 3 MODELS \\
0.25 & 0.34 & 0.16 & 0.15 \\
\hline
\end{tabular}

the observed discharges during $3 \mathrm{~h}$ before $t_{0}$. The output of the networks is the discharge $6 \mathrm{~h}$ after the $t_{0}$. Summarizing, the data have been divided in three groups using the SOM, in order to identify three different hydrological states of the system, and each group has been calibrated with a Feed Forward Network in order to forecast the discharge $6 \mathrm{~h}$ in advance. Moreover, to avoid the risk of overfitting the calibration data, an early stopping procedure has been used introducing a verification data set, included between 1 June 1997 and 31 January 1998. This procedure stops the Neural Network calibration as soon as the evaluation indexes computed on the verification data set starts to decrease. Finally, the data included between 1 February 1998 and 30 September 2002 have been used for validating the model.

In order to make coherent the forecasts of each model also the TETIS and TOPKAPI models have been used to predict the discharge $6 \mathrm{~h}$ in advance, assuming, as done with the ANN, that the precipitation is null during the forecast time.

In Fig. 10 a schematic summary of the division of the data used for calibrating and validating each model is depicted.

The two physically based models are conceptually quite similar; it can be highlighted that the TOPKAPI model tends to underestimate the highest flood events, to overestimate the smallest ones and to reproduce the flood events of medium magnitude quite well. The TETIS model also generally underestimates the highest events and often underestimates the small events too. The ANN model, due to its nature of data driven model, is not able to well reproduce the peak flows, which are often underestimated and predicted with a delay of 1 or $2 \mathrm{~h}$, but it perfectly reproduces the low flows.

\subsubsection{Predictive uncertainty assessment}

The MCP is applied in three phases and Joint TNDs have been used in each phase.

1. In the first step, each model is processed separately. All the historical data are being processed and the expected value of the predictand conditional to a single model is computed at each time step from the predictive distribution. Figure $11 \mathrm{a}, \mathrm{b}$ and $\mathrm{c}$, schematically represents the predictive distribution computed separately with each model. For the ANN model it was not necessary to divide the data in two samples because the joint distribution of observed and forecasted transformed values was well represented by just a single bi-variate normal distribution. The TNDs have been used for the other two models and both of them provide a lower uncertainty for the upper sample.

2. In the second step, the series of the expected values of the predictand conditional on each model forecast is processed with the MCP multivariate approach and the combined expected value of the predictand conditional to all the models is computed at each time step from the predictive distribution.

3. In the third step, the series of expected values of the predictand conditional to all the models is finally processed in order to properly estimate the predictive density. This last step is required, as it will be discussed in the next section, due to the non perfect agreement between the empirical density of residual and the assumed Normal distribution.

Figure 12a and $\mathrm{b}$ summarizes the obtained results with regard to the models combination computed from the expected value of the predictive distribution. Figure 12a represents the standard error and Fig. 12b represents the Nash-Sutcliffe coefficient.

In Figs. 13, 14 and 15, 16 two examples of models combination are shown, one during the calibration period and the other one during the validation period. In both events the uncertainty band gets narrower as the number of models increases and in the calibration event the expected value computed with the combination of all the models well matches the observed series. In the validation event, the pick flow is quite better represented when only the TOPKAPI model is used, probably due to its better forecast in this specific case, but also in this event the uncertainty band is reduced combining all the models.

The combination of the three models' predictions, obtained by assigning different weights to each model according to the Bayesian theory, allows the forecast quality to be improved as shown by the evaluation indexes in Fig. 12a and $b$. The two physically based model structures are very similar, so this leads to a little gain in terms of forecast improvement, represented by the standard deviation of the errors and the Nash-Sutcliffe efficiency index (Fig. 12a and b). On the contrary, the combination of one physically based model with the data driven model leads to greater improvements in forecast and, in particular, the combination of all the three models gives the best values of the analyzed indexes (Fig. 12a and b).

The knowledge of the uncertainty distribution also allows the probability of exceeding an alert threshold to be estimated, that is a stochastic way to predict the flooding risk. The threshold has been set at $350 \mathrm{~m}^{3} \mathrm{~s}^{-1}$. In Figs. 17 and 18 , the comparison between the deterministic and stochastic discharge forecasts and the correspondent probability of exceeding the threshold is shown. 


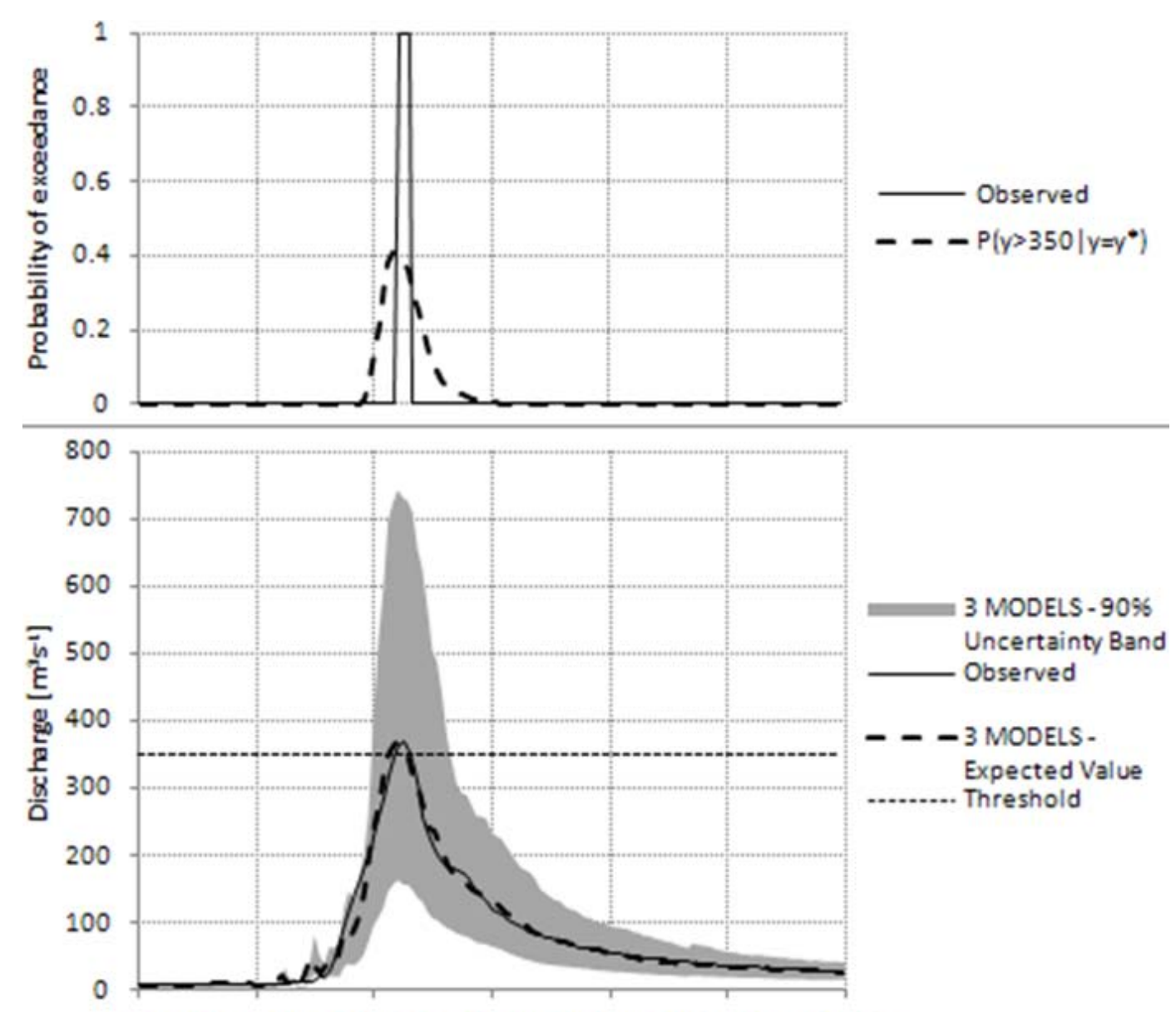

15/12/01 16/12/0117/12/01 18/12/01 19/12/0120/12/01 21/12/01

Fig. 17. Flood event for the calibration period. The lower panel represents the discharge forecast; observed values (continuous line); expected value conditioned to the TOPKAPI, TETIS and ANN forecasts (dashed line); $90 \%$ uncertainty band (grey area); alarm threshold of $350 \mathrm{~m}^{3} \mathrm{~s}^{-1}$ (small dashed line). The upper panel represents the probability of exceeding the alarm threshold; observed binary response (continuous line) and Probability of exceeding the threshold computed by the MCP (dashed line).

It has been also shown that the combination of several models leads to improved estimation of such exceeding probability. Tables 1 and 2 exemplify the improvements obtainable by the Bayesian combination of the different models. Table 1 concurs with the behaviour represented in Fig. 1 showing the probability that the true value exceeds the $350 \mathrm{~m}^{3} \mathrm{~s}^{-1}$ threshold when the expected value of prediction equals $250 \mathrm{~m}^{3} \mathrm{~s}^{-1}$, computed for each model and their Bayesian combination. One can see the reduction of exceedance probability as a function of the quality of the forecast. Finally, the effect of the introduction of the probabilistic forecast approach can be appreciated in Table 2. It shows, similarly to what is qualitatively displayed in Fig. 2, the expected value of the prediction corresponding to the probability of $20 \%$ to exceed the $350 \mathrm{~m}^{3} \mathrm{~s}^{-1}$ threshold; this value is computed for each model and for their Bayesian combination. As can be seen better models allow to wait until the expected value of prediction is closer to the flooding
Table 2. Expected value of prediction corresponding to the probability of $20 \%$ that the true value will exceed the $350 \mathrm{~m}^{3} \mathrm{~s}^{-1}$ threshold, computed for each model and their Bayesian combination.

\begin{tabular}{llcc}
\hline \multicolumn{4}{c}{$E[y \mid \hat{y}] \mid\left[P\left(y>350 \mathrm{~m}^{3} \mathrm{~s}^{-1} \mid \hat{y}\right)=0.2\right]$} \\
\hline TOPKAPI & TETIS & ANN & 3 MODELS \\
$217 \mathrm{~m}^{3} \mathrm{~s}^{-1}$ & $138 \mathrm{~m}^{3} \mathrm{~s}^{-1}$ & $270 \mathrm{~m}^{3} \mathrm{~s}^{-1}$ & $284 \mathrm{~m}^{3} \mathrm{~s}^{-1}$ \\
\hline
\end{tabular}

level, while worse models require earlier action corresponding to lower levels on the basis of the principle of precaution, which corresponds to the fact that the decision maker is more uncertain. 


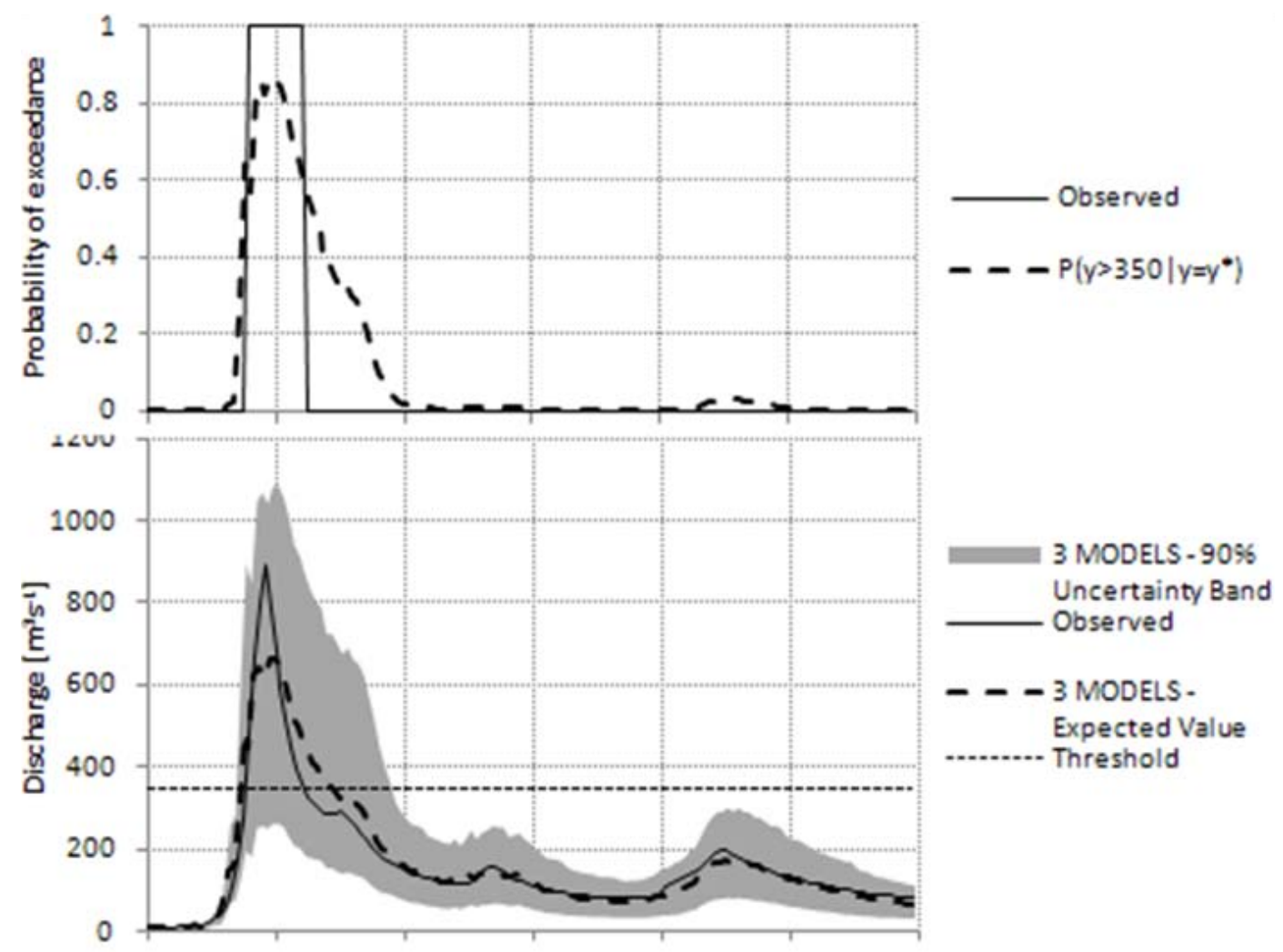

04/01/98 05/01/98 06/01/98 07/01/98 08/01/98 09/01/98 10/01/98

Fig. 18. Flood event for the validation period. The lower panel represents the discharge forecast; observed values (continuous line); expected value conditioned to the TOPKAPI, TETIS and ANN forecasts (dashed line); $90 \%$ uncertainty band (grey area); alarm threshold of $350 \mathrm{~m}^{3} \mathrm{~s}^{-1}$ (small dashed line). The upper panel represents the probability of exceeding the alarm threshold; observed binary response (continuous line) and Probability of exceeding the threshold computed by the MCP (dashed line).

\subsubsection{Quantiles assessment}

As mentioned in the previous section, in order to obtain a more adherent representation of the predictive density, which is essential for decision making, a third step was deemed necessary in the procedure after analyzing the residuals of the second step. The probability distribution of these residuals, although appearing reasonably well represented by a Normal distribution in the central portion, showed high kurtosis values due to fatter tails, which induced overestimating the predictive variance under the Normal Distribution assumption. Due to the fact that decisions in flood management are essentially based on probabilities in the range $0.1-0.9$ (one must realize that 0.9 probability of overtopping a threshold is already extremely high when taking decisions) it was decided that the estimation of the full predictive density would be based on a reduced set of data: namely all the couples of observation-expected value of the predictand conditional to all the models, that would generate a residual falling into the probability range $0.1-0.9$. Therefore, in the third step, the application of MCP was based on this reduced set of data only, and the results were quite rewarding even in the case of the Baron Fork river example where the three forecasting models were not extremely accurate. Figure 19 shows the results for the Baron Fork river example in the Normal space in terms of probability densities of residuals and of the assumed Normal predictive density, for the calibration and the verification periods. Please note that the empirical densities of residuals displayed in Fig. 19 are obtained with all the observations, not limited to the reduced set used in the estimation phase. It can be noted that apart from the upper and lower tails the probability density is correctly reproduced both for the calibration (left) and verification (right) periods. Figure 20 is the most important verification tool for establishing the correctness of the approach; it shows the comparison for the Baron Fork river example between the predicted probability of being in a range around the expected value $(10 \%$, $20 \%, . ., 90 \%$ ) and the corresponding empirical probability estimated from the observations both for the calibration (left) and verification (right) periods. Again it is possible to note that the approach seems to correctly estimate the predictive density. 
Residuals Distribution - Calibration

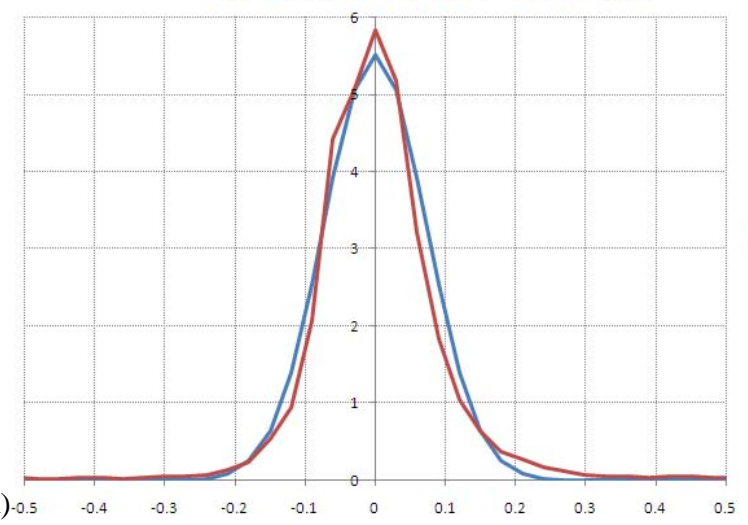

Residuals Distribution - Verification

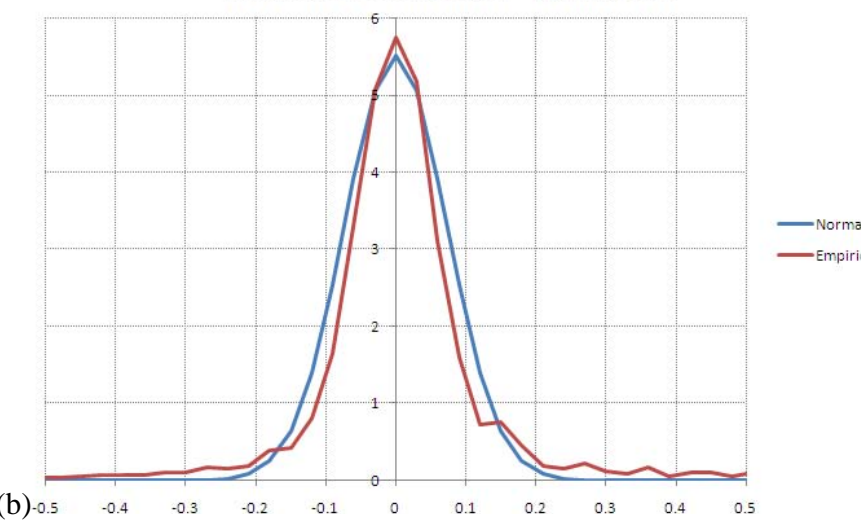

Fig. 19. Comparison between the empirical distribution of residuals and the assumed Normal distribution $(\sigma=0.072)$. The results were obtained considering the entire calibration (a) and verification (b) periods for the bayesian combination of the three models.

\section{Conclusions}

This paper is focused on the Model Conditional Processor (Todini, 2008) development for assessing predictive uncertainty. Two applications, the first one to the Po River (Italy) and the second one to the Baron Fork River (OK, USA), allowed to draw some important conclusions, which are summarized below.

The predictive uncertainty assessment starts with the identification of the marginal distributions of the observed and predicted data as well as their joint distribution. Such marginal distributions are often unknown in the untransformed observation space, and moreover it is extremely difficult to make hypotheses on the shape of their joint distribution. Several works in the literature (Krzysztofowicz, 1999; Montanari and Brath, 2004; Todini, 2008) suggested to use a non-parametric approach based on order statistics, namely to use the Weibull Plotting Position as an estimate of the probability of an ordered vector. Accordingly, a nonlinear transformation, the Normal Quantile Transform, is used to move from the original observation space to the Normal one, where
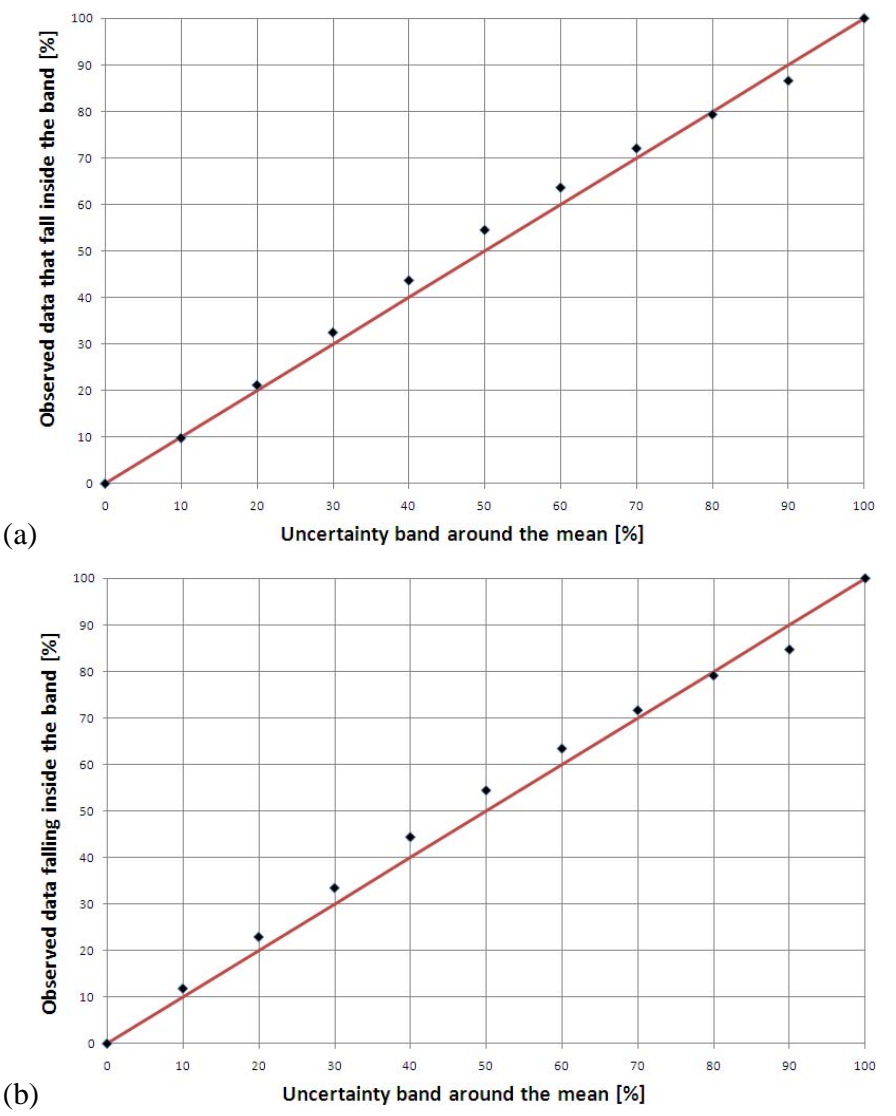

Fig. 20. Percentage of observed data that fall inside the uncertainty band at various probability levels defined with a $10 \%$ interval. The red line represents the perfect behaviour. The results were obtained considering the entire calibration (a) and verification (b) periods for the bayesian combination of the three models.

by construction the marginal distributions assume a Standard Normal shape and the joint distribution can be reasonably approximated by a Multivariate Normal distribution. Nonetheless, this approach has some disadvantages. First of all, it implies to identify additional models to adjust the quantiles outside the range of the historical available data. The proposed technique is quite sensitive to the shape and to the parameters of these models and some precautions in the choice of the subset of observations used for calibrating the tails data must be taken. They must contain a large variety of cases, as required by any Bayesian approach, and in order to reduce the uncertainty on the marginal distribution tails the calibration data must include the highest number of extreme cases.

The assumption of a Normal Multivariate joint distribution in the transformed space implies unavoidable approximations and it does not account for the error heteroscedasticity. In order to reduce it a non-linear regression model could be used. In this paper a piecewise linear approach has been preferred to a fully non-linear model. The piecewise linear approach allows for the use of Truncated Multivariate 
Normal joint distributions. This technique can be easily developed and applied obtaining good results such as for the study cases where it has been used. The results shown in Fig. 20 demonstrate that the joint distribution is well represented with this technique, even if some unavoidable approximations are still present. Nevertheless, the methodology should be tested considering other catchments with different features and for each specific application the correctness of the joint distribution representation must be verified. However, it must be noted that the use of the TNDs does not affect those cases when the data are homoscedastically distributed, as shown in Figs. 8 and 11c, but it helps to take into account the heteroscedasticity when it is present, as shown in Fig. 11a and b.

Nevertheless, the TND assumption for the joint distribution showed to be not fully correct; in fact, under the hypothesis of Normality, the residuals should be distributed according to a Normal distribution. In the case of the Baron Fork river, they showed to be Normally distributed in the central portion, but also to have a high kurtosis due to their fat tails. This problem can be reasonably solved in the last phase of the MCP application taking into account just the data that provide residuals inside the $0.1-0.9$ probability band. Figures 19 and 20 confirmed the correctness of PU assessment, at least for probability values included inside the $80-90 \%$ around the expected value of the predictand.

Multiple predictions originated by several models, as discussed in the introduction, is of difficult understanding and interpretation by the decision makers. The application of the MCP to the Baron Fork river has shown that this technique allows the correct combination of different forecasts into an unique probability of the event, which is of much easier interpretation and use in the decision making process. Moreover, the obtained results show that the combination of models of different nature allows the probabilistic forecast to improve the deterministic forecast of each model, taking advantage of the benefits of different hydrological approaches.

With this work, a discussion about the convenience of using a probabilistic threshold instead of a deterministic one in order to estimate the flooding risk and help the decision making process about giving or not a flood alarm, has been initiated. When hydrological forecasts can be defined in terms of a binary response (i.e. being below or above a threshold or giving or not a flood alarm) the probabilistic threshold concept allows the reliability and the information provided by different models to be taken into account in a combined and unique probability level. Therefore, the emergency manager can express his/her propensity to the risk in terms of probability of flooding and not just comparing a pre-fixed real threshold with the model forecast (which is nothing else than virtual reality), as usually done with the deterministic approach. In this respect, the paper also highlighted the need for a change in flood forecasting and warning approaches with the definition of probabilistic thresholds which aim at taking advantage of probabilistic forecasts in a more effective way. The results presented in Sect. 4.2.4 show the good performance of the methodology at correctly assessing the quantiles up to 80-90\% around the expected value of the predictand, which then allows a decision maker to correctly infer the probability of exceeding an alarm threshold or a dyke.

Acknowledgements. This work was supported by the Italian Ministry of Education. The authors thank C. Mazzetti and M. Martina for their guidance and advices. G. C. would like to thank J. C. Munera and F. Francés for providing the results and explanations of the TETIS model. G. C. also thanks L. Pujol and Hidrogaia S. L. for the support in developing the Artificial Neural Networks Model. Finally, the authors thank the Civil Protection of Emilia Romagna Region and the NOAA's National Weather Service for providing the data used in the study cases.

Edited by: K. Bishop

\section{References}

De Groot, M. H.: Optimal Statistical Decision, McGraw-Hill, New York, 1970.

Dempster, A. P., Laird, N. M., and Rubin, D. B.: Maximum likelihood from incomplete data via the EM algorithm, J. Roy. Stat. Soc. B, 39, 1-39, 1977.

Di Baldassarre, G. and Montanari, A.: Uncertainty in river discharge observations: a quantitative analysis, Hydrol. Earth Syst. Sci., 13, 913-921, doi:10.5194/hess-13-913-2009, 2009.

Dottori, F., Martina, M. L. V., and Todini, E.: A dynamic rating curve approach to indirect discharge measurement, Hydrol. Earth Syst. Sci., 13, 847-863, doi:10.5194/hess-13-847-2009, 2009.

Francés, F., Velez, J. I., and Velez, J. J.: Split-parameter structure for the automatic calibration of distributed hydrological models, J. Hydrol., 332, 226-240, 2007

Kalman, R. E.: A new Approach to linear filtering and prediction problems. J. Basic Eng. Trans. ASME, 82 D, 35-45, 1960.

Kalman, R. E. and Bucy, R. S.: New results in linear filtering and prediction theory. J. Basic Eng. Trans. ASME, 83 D, 95-108, 1961.

Kennedy, M. C. and O'Hagan, A.: Bayesian calibration of computer models, J. Roy. Stat. Soc. B., 63, 425-450, 2001.

Koenker, R.: Quantile Regression, Econometric Society Monographs, Cambridge University Press, New York, NY, 2005.

Kohonen T.: The self-organizing map, P. IEEE, 78, 1464-1480, doi:10.1109/5.58325, 1990.

Krzysztofowicz, R.: Bayesian theory of probabilistic forecasting via deterministic hydrologic model, Water Resour. Res., 35, 2739-2750, 1999.

Krzysztofowicz, R. and Kelly, K. S.: Hydrologic uncertainty processor for probabilistic river stage forecasting, Water Resour. Res., 36, 3265-3277, 2000.

Liu, Z. and Todini, E.: Towards a comprehensive physically-based rainfall-runoff model, Hydrol. Earth Syst. Sci., 6, 859-881, doi:10.5194/hess-6-859-2002, 2002.

Mardia, K. V., Kent, J. T., and Bibby, J. M.: Multivariate Analysis. Probability and Mathematical Statistics, Academic Press, London, 1979. 
Montanari, A. and Brath, A.: A stochastic approach for assessing the uncertainty of rainfall-runoff simulation, Water Resour. Res., 40, W01106, doi:10.1029/2003WR002540, 2004

Parker, D. B.: Optimal algorithms for adaptive networks: Second order backpropagation, second order direct propagation and second order Hebbian learning, IEEE 1st Int. Conf. Neural Networks, 2, 593-600, 1987.

Pujol, L.: Prediccion de caudales en tiempo real en grandes cuencas utilizando redes neuronales artificiales, Ph.D. dissertation, Polytechnic University of Valencia, Department of Hydraulic Engineering and Environment, 34-39, 126-134, 2009.

Raftery, A. E.: Bayesian model selection in structural equation models, in: Testing Structural Equation Models, edited by: Bollen, K. A. and Long, J. S., Sage, Beverly Hills, CA, 163-180, 1993.

Raftery, A. E., Balabdaoui, F., Gneiting, T., and Polakowski, M.: Using Bayesian model averaging to calibrate forecast ensembles, Mon. Weather Rev., 133, 1155-1174, 2005.

Raiffa, H. and Schlaifer, R.: Applied Statistical Decision Theory, The MIT Press, Cambridge, 1961.

Tallis, G. M.: The moment generating function of the truncated multi-normal distribution, J. Roy. Stat. Soc. B, 23, 223-229, 1961.

Todini E.: Mutually Interactive State/Parameter Estimation (MISP), in: Application of Kalman Filter to Hydrology, edited by: ChaoLin Chiu, Hydraulics and Water Resources, University of Pittsburgh, Penn, 1978.

Todini, E.: A model conditional processor to assess predictive uncertainty in flood forecasting, Int. J. River Basin Manage., 6, 123-137, 2008.

Todini, E.: Predictive uncertainty assessment in real time flood forecasting, edited by: Baveye, P. C., Laba, M., and Mysiak, J., Uncertainties in Environmental Modelling and Consequences for Policy Making, NATO Science for Peace and Security Series C: Environmental Security, Springer Netherlands, Amsterdam, NL, 205-228, doi:10.1007/978-90-481-2636-1_9, 2009.

Todini, E. and Bossi, A.: PAB (Parabolic and Backwater) an Unconditionally Stable Flood Routing Scheme Suited for Real-Time Forecasting and Control, J. Hydraul. Res., 24, 405-424, 1986.
Todini, E. and Ciarapica, L.: The TOPKAPI model, in: Mathematical Models of Large Watershed Hydrology, chap. 12, edited by: Singh. V. P., Water Resources Publications, Littleton, 2001.

Van der Waerden, B. L.: Order tests for two-sample problem and their power I, Indagat. Math., 14, 453-458, 1952.

Van der Waerden, B. L.: Order tests for two-sample problem and their power II, Indagat. Math., 15, 303-310, 1953a.

Van der Waerden, B. L.: Order tests for two-sample problem and their power III, Indagat. Math., 15, 311-316, $1953 \mathrm{~b}$.

Vélez, J. J., Puricelli, M., López Unzu, F., and Francés, F.: Parameter extrapolation to ungauged basins with a hydrological distributed model in a regional framework, Hydrol. Earth Syst. Sci., 13, 229-246, doi:10.5194/hess-13-229-2009, 2009.

Vrugt, J. A. and Robinson, B. A.: Treatment of uncertainty using ensemble methods: Comparison of sequential data assimilation and Bayesian model averaging, Water Resour. Res., 43, W01411, doi:10.1029/2005WR004838, 2007.

Vrugt, J. A., Gupta, H. V., Bouten, W., and Sorooshian, S.: A Shuffled Complex Evolution Metropolis Algorithm for optimization and uncertainty assessment of hydrological model parameters, Water Resour. Res., 39, 1201, doi:10.1029/2002WR001642, 2003.

Weerts, A. H., Winsemius, H. C., and Verkade, J. S.: Estimation of predictive hydrological uncertainty using quantile regression: examples from the National Flood Forecasting System (England and Wales), Hydrol. Earth Syst. Sci., 15, 255-265, doi:10.5194/hess-15-255-2011, 2011.

Werbos, P.: Beyond Regression: New Tools for Prediction and Analysis in the Behavioral Science, Ph. D. dissertation, Harvard University, Cambridge, 1974.

Werbos, P.: Generalization of backpropagation with application to a recurrent gas model, Neural Networks, 1, 339-356, 1988.

Werbos, P.: Backpropagation through time: What it does and how to do it, P. IEEE, 78, 1550-1560, 1990. 